SLAC-PUB-4574

LBL-25018

February 1988

(M)

\title{
BOSE-EINSTEIN CORRELATIONS IN $e^{+} e^{-}$COLLISIONS*
}

\section{Juricic, ${ }^{(a)}$ G. Goldhaber, G. Gidal,}

G. Abrams, D. Amidei, (b) A. R. Baden, (c) J. Boyer, F. Butler, W. C. Carithers, M. S. Gold, L. Golding, ${ }^{(d)}$ J. Haggerty, ${ }^{(e)}$ D. Herrup, ${ }^{(f)}$ J. A. Kadyk, M. E. Levi, M. E. Nelson, ${ }^{(g)}$ P. C. Rowson, ${ }^{(a)}$ H. Schellman, ${ }^{(b)}$ W. B. Schmidke, P. D. Sheldon, ${ }^{(h)}$ G. H. Trilling, and D. R. Wood ${ }^{(i)}$

\section{Lawrence Berkeley Laboratory and Department of Physics, University of California, Berkeley, California 94720}

T. Barklow, A. Boyarski, M. Breidenbach, P. Burchat, ${ }^{(j)}$ D. L. Burke, D. Cords, J. M. Dorfan, G. J. Feldman, L. Gladney, ${ }^{(k)}$ G. Hanson, K. Hayes, D. G. Hitlin, ${ }^{(g)}$ R. J. Hollebeek, ${ }^{(k)}$ W. R. Innes, J. A. Jaros, D. Karlen, S. R. Klein, A. J. Lankford, R. R. Larsen, B. W. LeClaire, N. S. Lockyer, ${ }^{(k)}$ V. Lüth, R. A. Ong, M. L. Perl, B. Richter, K. Riles, M. C. Ross, R. H. Schindler, and J. M. Yelton ${ }^{(l)}$

\section{Stanford Linear Accelerator Center, Stanford University, Stanford, California 94905}

\section{T. Schaad $(m)$ and R. F. Schwitters \\ Harvard University, Cambridge, Massachusetts 02138}

* This work was supported in part by the United States Department of Energy, contract numbers DE-AC03-76SF00098 (LBL), DE-AC03-76SF00515 (SLAC), and DE-AC02-76ER03064 (Harvard).

(a) Present address: Columbia University, New York, NY 10027.

(b) Present address: University of Chicago, Chicago, IL 60637.

(c) Present address: Harvard University, Cambridge, MA 02138

(d) Present address: Therma-Wave Corp., Fremont, CA 94539.

(e) Present address: Brookhaven National Laboratory, Upton, NY 11973.

(f) Present address: Fermi National Laboratory, Batavia, IL 60510.

(g) Present address: California Institute of Technology, Pasadena, CA 91125.

(h) Present address: University of Illinois, Urbana, IL 61801.

(i) Present address: CERN, CH-1211, Geneva 23, Switzerland.

(j) Present address: University of California, Santa Cruz, CA 95064.

(k) Present address: University of Pennsylvania, Philadelphia, PA 19104.

(l) Present address: University of Florida, Gainesville, FL 32611.

(m) Present address: University of Geneva, CH-1211, Geneva 4, Switzerland. 


\begin{abstract}
Abstráct
Four data sets collected with the MARK II detector during its operation at the SPEAR and PEP $e^{+} e^{-}$storage rings are used to study the Bose-Einstein correlation between pairs and triplets of like sign charged pions. The data sets represent four different regions of energy available for hadron production: the $J / \psi$ at $\sqrt{s}=3.095 \mathrm{GeV}$, typical hadronic energy of $\approx 5 \mathrm{GeV}$ in two-photon events, and annihilation in the energy regions $\sqrt{s}=4-7 \mathrm{GeV}$ above the $J / \psi$, and $\sqrt{s}=29 \mathrm{GeV}$. The Bose-Einstein correlation is studied as a function of $Q^{2}$, the four-momentum difference squared of the pair, and of $Q_{3}^{2}$, an analogous quantity defined for triplets. After corrections for Coulomb effects and pion misidentification, pair analyses indicate a nearly full Bose-Einstein enhancement $(\lambda \approx 1)$ in the $J / \psi$ and the two-photon data, and about half the maximum value in the two higher energy data sets. The pair analysis parameter $r$ lies within a band of $\pm 0.1 \mathrm{fm}$ around $0.7 \mathrm{fm}$ and is essentially the same for all four data sets. Pion triplet analyses give consistent results for the triplet parameters $\lambda_{3}$ and $r_{3}$. In an attempt to investigate the shape of the pion source, we also study the Bose-Einstein correlation in pion pairs using two-dimensional distributions in components of $Q^{2}$.
\end{abstract}


Table of Contents

I. INTRODUCTION

II. DATA ACQUISITION AND REDUCTION . . . . . . 8

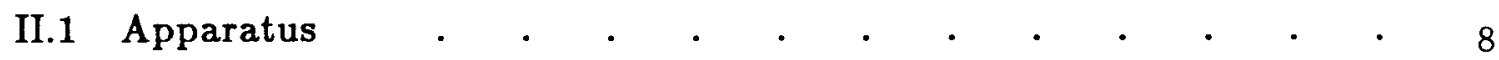

II.2 Data Acquisition . . . . . . . . . . . . .

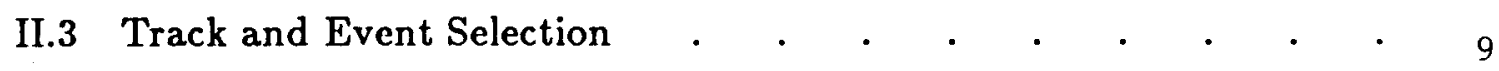

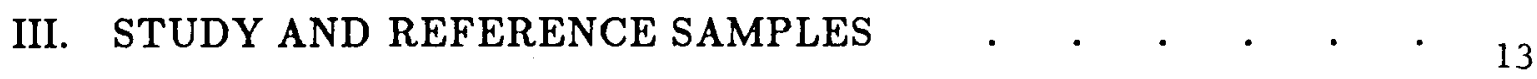

IV. CORRECTIONS.$\quad \cdot \quad \cdot \quad \cdot \quad \cdot \quad \cdot \quad \cdot \quad \cdot \quad \cdot \quad \cdot \quad \cdot{ }_{21}$

IV.1 Final State Interactions $\quad \cdot \quad \cdot \quad \cdot \quad \cdot \quad \cdot \quad \cdot \quad \cdot \quad \cdot \quad \cdot 21$

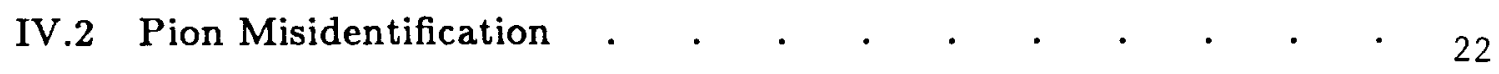

IV.3 Drift Chamber Performance - . . . . . . . 22

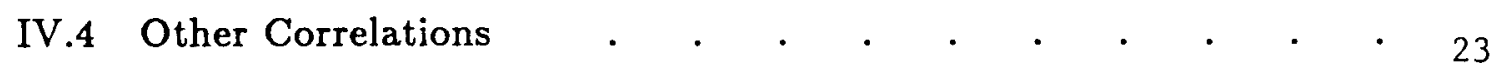

V. RESULTS AND DISCUSSION .

V.1 Calculation and Fitting of the Correlation Function . . $\quad 25$

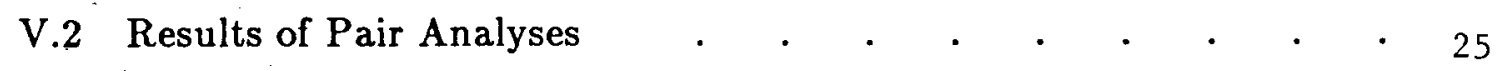

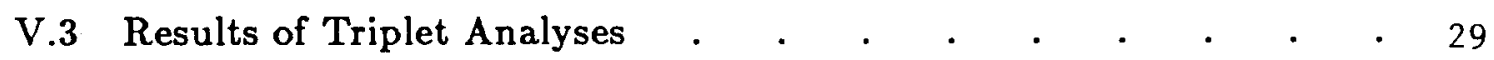

V.4 Bose-Einstein Correlation in PEP $\gamma \gamma$ Pairs . . . . . $\quad 32$

V.5 Bose-Einstein Correlation in Components of $Q^{2}$. . . . 33

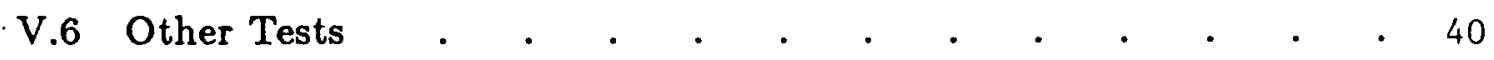

V.7 The Effect of Long Lived Resonances and Particles • . . 42

VI. SUMMARY AND CONCLUSIONS . $\cdot$. $\cdot$. $\cdot$. 45

Appendix A. COULOMB CORRECTION . . . . . . $\quad 51$

Appendix B. PION MISIDENTIFICATION CORRECTION • • $\quad 55$

Appendix C. MIXED CLUSTER REFERENCE SAMPLES • • • 57

REFERENCES • • • • • • • • • • • • 59 


\section{INTRODUCTION}

An enhancement in the production of pairs of pions of like charge and similar momentum has been observed in a variety of experiments ${ }^{1}$, including hadronic reactions $s^{2,3,4}$, heavy-ion collisions ${ }^{5,6}, e^{+} e^{-}$annihilation $^{7,8,9}$, two photon collisions ${ }^{10}$, and $\mu$-hadron collisions ${ }^{11}$. This phenomenon, known as the Bose-Einstein correlation or the GGLP effect, was originally noted in $p \bar{p}$ annihilation ${ }^{12}$ and was interpreted as a consequence of Bose-Einstein statistics obeyed by like-charged pion pairs $^{13}$. In analogy with the work of Hanbury-Brown and Twiss ${ }^{14}$ in stellar intensity interferometry, the Bose-Einstein correlation is described in terms of a correlation function $C_{2}$, which is defined as the ratio of the joint production probability $P\left(k_{1}, k_{2}\right)$ to the product $P\left(k_{1}\right) P\left(k_{2}\right)$ of single pion production probabilities, where $k_{1}$ and $k_{2}$ are the pion four-momenta. For a chaotic (thermal) pion source, the correlation function is given by

$$
C_{2}\left(k_{1}, k_{2}\right) \equiv \frac{P\left(k_{1}, k_{2}\right)}{P\left(k_{1}\right) P\left(k_{2}\right)}=1+\left|\hat{\rho}\left(k_{1}-k_{2}\right)\right|^{2},
$$

where $\hat{\rho}\left(k_{1}-k_{2}\right)$ is the Fourier transform of the source distribution. The symmetrization requirement on the pion pair wave function results in the interference term $\left|\hat{\rho}\left(k_{1}-k_{2}\right)\right|^{2}$, which contains information about the space-time structure of the pion source ${ }^{15,16,17}$.

Since, in typical hadron production experiments it is not feasible to accurately calculate inclusive production distributions $P\left(k_{1}\right)$, and $P\left(k_{2}\right)$, one studies the BoseEinstein correlation by comparing the distribution of like charged pion pairs with a distribution of reference sample pairs which are free of the Bose-Einstein correlation. The ratio $R$ of the like pair distribution divided by, a suitably normalized, reference pair distribution is parametrized in terms of a Gaussian function of the four-momentum difference squared $Q^{2}$,

$$
\begin{gathered}
R\left(Q^{2}\right)=1+\lambda e^{-r^{2} Q^{2}}, \\
Q^{2}\left(k_{1}, k_{2}\right) \equiv-\left(k_{1}-k_{2}\right)^{2}=M_{12}^{2}-4 m^{2}
\end{gathered}
$$


where $M_{12}$ is the invariant mass of the pair, $m$ is the pion mass, and $\lambda$ and $r$ are parameters determined by a fit to the data. Although this is an empirical expression, it has been shown to describe $e^{+} e^{-}$collision data very well ${ }^{7,8,9}$ over a wide range of center of mass energies.

In the limit of identical momenta, a completely chaotic source is expected to produce $R\left(Q^{2} \rightarrow 0\right)=2$. Since most experiments measure a less than maximum Bose-Einstein enhancement, the parameter $\lambda$ was introduced ${ }^{2}$ in front of the enhancement term to represent the strength of the observed effect. For a fully coherent pion source (pion laser), the Bose-Einstein enhancement term is expected to vanish $(\lambda=0)$, and claims have been made that $\lambda$ is thus a measure of the degree of source coherence ${ }^{18,19}$. Bowler ${ }^{20}$ points out, however, that this interpretation may be overly simplistic in high energy $e^{+} e^{-}$collisions, and that a variety of source conditions can produce $\lambda<1$.

Interpretation of the parameter $r$ is also the subject of some debate. In the case of a fully chaotic source, the parameter $r$ corresponds to an average over the spatial and temporal source dimensions. Current models of the space-time evolution of particle production present a very different picture of the hadronic source. In the case of $e^{+} e^{-}$jets, for example, QCD leads us to expect that the pions are produced along a color string ${ }^{21}$, and at PEP energies $(\sqrt{s}=29 \mathrm{GeV})$ the longitudinal extent of pion production points is expected to be on the order of $30 \mathrm{fm}$. This is in contrast to the typical measured values of $r \approx 0.7 \mathrm{fm}$ in $e^{+} e^{-}$experiments. In addition, most experiments have found that the simple Gaussian form in $Q^{2}$ gives the best fit to the Bose-Einstein enhancement in the data. It has been pointed out ${ }^{22}$, however, that since pion momenta in the color string model are correlated with the production region along the string, the parameter $r$ may be a measure of the size of the local emitting region rather than the extent of the entire source. This would reconcile the string model with the observation of an apparently "spherical" source. In fact, the classical Artru-Mennessier string model has been modified to include the Bose-Einstein correlation ${ }^{21,23}$ and has been shown to successfully reproduce the major features of the Bose-Einstein enhancement observed in the data 24 . While 
the debate over the interpretation of $r$ continues, in this analysis we will use $r$ as a bench mark for the relative comparison of data sets and experiments.

The analysis of the Bose-Einstein correlation in like charged pion triplets follows the procedure for pairs. The triplet correlation function for a chaotic source is given by

$$
\begin{aligned}
C_{3}\left(k_{1}, k_{2}, k_{3}\right) & =1+\left|\hat{\rho}\left(k_{1}-k_{2}\right)\right|^{2}+\left|\hat{\rho}\left(k_{2}-k_{3}\right)\right|^{2}+\left|\hat{\rho}\left(k_{3}-k_{1}\right)\right|^{2} \\
+ & 2 \operatorname{Re}\left|\hat{\rho}\left(k_{1}-k_{2}\right) \hat{\rho}\left(k_{2}-k_{3}\right) \hat{\rho}\left(k_{3}-k_{1}\right)\right|
\end{aligned}
$$

where the last term is referred to as the purely triplet enhancement. If we naively use the pair parameterization

$$
\left|\rho\left(k_{i}-k_{j}\right)\right| \approx \sqrt{\lambda} e^{-r^{2} Q_{i j}^{2} / 2}
$$

where $Q_{i j}^{2} \equiv Q^{2}\left(k_{i}, k_{j}\right)$, and if we neglect phase factors ${ }^{9}$, the triplet correlation function can be expressed as

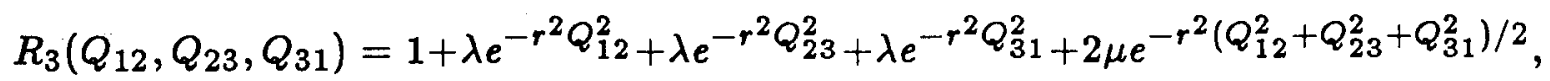

where $\mu$ represents the strength of the purely triplet enhancement term $0<\mu<1$. Limited statistics make it impossible to study $R_{3}$ as a function of the three variables $Q_{12}, Q_{23}$, and $Q_{31}$, so $R_{3}$ is usually parameterized ${ }^{25}$ in analogy with pair analysis

$$
R_{3}\left(Q_{3}^{2}\right)=1+\lambda_{3} e^{-r_{3}^{2} Q_{3}^{2}}
$$

as a function of the single variable

$$
Q_{3}^{2}\left(k_{1}, k_{2}, k_{3}\right) \equiv M_{123}^{2}-9 m^{2}=Q_{12}^{2}+Q_{23}^{2}+Q_{31}^{2}
$$

where $M_{123}$ is the invariant mass of the three pions. In the limit of small $Q_{12}^{2} \approx$ $Q_{23}^{2} \approx Q_{31}^{2} \equiv Q_{i j}^{2}, Q_{3}^{2} \approx 3 Q_{i j}^{2}$, and self-consistency between the pair and triplet parameterizations leads us to expect that $\lambda_{3} \approx 3 \lambda+2 \mu<5$ and $\left(r^{2} / 3\right)<r_{3}^{2}<\left(r^{2} / 2\right)$. 
Although we cannot make a significant measurement of $\mu$ within our statistics, we find that the remaining parameters satisfy these limits.

In an effort to probe the shape of the pion source, the Bose-Einstein correlation is also studied in terms of components of the four-momentum difference relative to some particular axis defined in the event. If we define $q_{0} \equiv\left|E_{1}-E_{2}\right|$ as the energy difference of the pair, $\vec{q} \equiv \vec{p}_{1}-\vec{p}_{2}$ as the three-momentum difference $(q \equiv|\vec{q}|)$, and $\hat{u}$ as a unit vector along the chosen axis, these variables are related to $Q^{2}$ as follows:

$$
\begin{gathered}
Q^{2}=q^{2}-q_{0}^{2}, \\
Q^{2}=q_{T}^{2}+q_{L}^{2}-q_{0}^{2}, \\
Q^{2}=q_{T}^{2}+q_{L^{\prime}}^{2},
\end{gathered}
$$

where

$$
\begin{gathered}
\vec{q}_{T} \equiv \vec{q} \times \hat{u} \\
q_{L} \equiv \vec{q} \cdot \hat{u} \\
q_{L^{\prime}}^{2} \equiv q_{L}^{2}-q_{0}^{2} .
\end{gathered}
$$

The unit vector $\hat{u}$ can be defined pair by pair as

$$
\hat{u} \equiv \frac{\vec{p}_{1}+\vec{p}_{2}}{\left|\vec{p}_{1}+\vec{p}_{2}\right|}
$$

which we refer to as the Kopylov axis ${ }^{26}$. In this case there is a simple relationship between $q_{L}$ and $q_{0}$

$$
q_{L}=q_{0} \frac{\gamma}{\sqrt{\gamma^{2}-1}}
$$

- where $\gamma$ is defined for the pion pairs as

$$
\gamma \equiv \frac{E_{1}+E_{2}}{M_{12}}
$$

Then

$$
q_{L^{\prime}}^{2}=\frac{q_{L}^{2}}{\gamma^{2}}=\frac{q_{0}^{2}}{\gamma^{2}-1}
$$


From these relations we can see that $q_{L^{\prime}}$ is the longitudinal component of the threemomentum difference in the pion pair rest frame. We also note that $q_{L} \approx q_{0}$.

In two jet events, $\hat{u}$ can also be defined event by event as the sphericity axis. In this case the relationship between $q_{L}$ and $q_{0}$ is no longer a simple function of $\gamma$. In either definition of $\hat{u}$, the variables $q_{T}$ and $q_{L^{\prime}}$ are Lorentz invariant for a boost along $\hat{u}$, while $q_{L}$ and $q_{0}$ are not.

These quantities are used in two alternative parameterizations of the BoseEinstein correlation. The first is a straightforward expansion of the familiar expression for $R\left(Q^{2}\right)$ and gives

$$
R\left(Q^{2}\right)=1+\lambda e^{-r_{T}^{2} q_{T}^{2}-r_{L}^{2} q_{L}^{2}+r_{0}^{2} q_{O}^{2}}=1+\lambda e^{-r_{T}^{2} q_{T}^{2}-r_{L^{\prime}}^{2} q_{L^{\prime}}^{2}}
$$

The second parameterization is based on the Kopylov-Podgoretskii model of a disk source $^{26}$, which can be approximated ${ }^{8}$ in our data by a Gaussian distribution of sources in space and time:

$$
R(\text { Kopylov })=1+\lambda e^{-r_{T}^{2} q_{T}^{2}-r_{0}^{2} q_{0}^{2}}
$$

Note that here in the Kopylov form, one obtains $-r_{0}^{2} q_{0}^{2}$ in the exponent, rather than the $+r_{0}^{2} q_{0}^{2}$ term obtained from an expansion of $Q^{2}$. Since we do not have enough statistics to study $R$ in terms of three variables, we effectively integrate over one at a time by considering the two-dimensional distributions

$$
\begin{aligned}
& R\left(q_{T}^{2}, q_{L}^{2}\right)=1+\lambda e^{-r_{T}^{2} q_{T}^{2}-r_{L}^{2} q_{L}^{2}} \\
& R\left(q_{T}^{2}, q_{0}^{2}\right)=1+\lambda e^{-r_{T}^{2} q_{T}^{2}-r_{0}^{2} q_{0}^{2}}
\end{aligned}
$$

as well as the distribution in the invariant quantities,

$$
R\left(q_{T}^{2}, q_{L^{\prime}}^{2}\right)=1+\lambda e^{-r_{T}^{2} q_{T}^{2}-r_{L^{\prime}}^{2} q_{L^{\prime}}^{2}}
$$

Using the parameterizations and variables discussed above, we investigate the Bose-Einstein correlation in four separate data sets, all produced in $e^{+} e^{-}$collisions. 
These represent four different regions of energy available for hadron production, as well as different production mechanisms.

1. One data set, SPEAR $J / \psi$, consists of hadronic events collected at $E_{c m}=$ $3.095 \mathrm{GeV}$, where the $J / \psi$ decays primarily via three gluons and partly through a virtual photon.

2. A second data set, PEP $\gamma \gamma$, is made up of two-photon events collected at $E_{c m}=29 \mathrm{GeV}$, where the average energy available for hadronization is $\approx 5 \mathrm{GeV}$. The production process in two-photon events is described by the Vector Dominance Model (VDM) when the hadrons are produced at low transverse momenta, while hard scattering is expected to dominate at high transverse momenta.

3. The third data set, SPEAR $q \bar{q}$, is composed of annihilations of $q \bar{q}$ into hadrons at $E_{c m}=4.1 \mathrm{GeV}$ to $6.7 \mathrm{GeV}$. In this continuum above the $\mathrm{J} / \psi$ and $\psi^{\prime}$, charm production is important, and jets become evident.

4. Annihilations of $q \bar{q}$ into hadrons at $E_{c m}=29 \mathrm{GeV}$ make up the last data set, PEP $q \bar{q}$. Here two jet production dominates, and three jet events make up nearly a quarter of the data. Both charm and bottom production are significant.

Our goal was to study how the parameters describing the Bose-Einstein correlation vary for these four different hadron production mechanisms. The data analyzed here was collected with a single detector, the MARK II, whose elements (relevant to this analysis) were basically unchanged during its running at SPEAR and PEP. Detector acceptance and efficiency were thus essentially the same for all data sets studied.

This paper is organized as follows. We describe the detector, the four data sets, and event selection in the next section. A discussion of study and reference samples in section three is followed in section four by a description of corrections applied to the data. Results and discussion are presented in section five. These include pair and triplet analyses, the special case of two photon data, pair analysis 
in terms of two parameters (components of $Q^{2}$ ), and some miscellaneous topics. The final section contains a summary and conclusions. 


\section{DATA ACQUISITION AND REDUCTION}

\section{1 APPARATUS}

The data sets used in this analysis were collected with the MARK II detector during its operation at the SPEAR (1978-79) and PEP (1980-84) $e^{+} e^{-}$collider storage rings located at the Stanford Linear Accelerator Center (SLAC). Reference 27 contains a detailed description of the detector in its SPEAR configuration. The major element of the detector upgrade for operation at PEP was the addition of a high-resolution vertex drift chamber ${ }^{28}$.

Central to this analysis is the measurement of charged particle momenta by the sixteen-layer cylindrical main drift chamber immersed in an axial magnetic field of $4.06 k G$ at SPEAR and $2.3 k G$ at PEP. The momentum resolution at SPEAR was $\left(\sigma_{p} / p\right)^{2}=(0.015)^{2}+(0.010 p)^{2}$, where $p$ is in $G e V / c$. In the PEP configuration the additional information from the vertex chamber partially offset the effects of a lower magnetic field, resulting in a net resolution of $\left(\sigma_{p} / p\right)^{2}=(0.025)^{2}+(0.011 p)^{2}$. The acceptance of the main drift chamber was $80 \%$ of $4 \pi$.

Except where noted, the MARK II components used for particle identification were the same in the two configurations. Forty eight strips of scintillator surrounded the drift chamber and formed the time of flight (TOF) system. The TOF resolution was about 300 ps at SPEAR, allowing good pion separation from kaons for momenta up to $1 \mathrm{GeV} / \mathrm{c}$ and from protons up to $2 \mathrm{GeV} / \mathrm{c}$. At PEP radiation damage to the scintillators degraded the TOF resolution to about $380 \mathrm{ps.} \mathrm{The} \mathrm{liquid-argon} \mathrm{elec-}$ tromagnetic calorimeter, used here to veto electrons, was a barrel of eight modules surrounding the TOF system and covering about $65 \%$ of $4 \pi$. The calorimeter was surrounded by four walls of the muon system, which consisted of (two at SPEAR -and four at PEP) layers of steel and proportional tubes and covered about $45 \%$ of $4 \pi$.

The small angle tagging (SAT) system, added to the MARK II in the PEP configuration, provided tracking and calorimetry at polar angles of $21 \mathrm{mr}$ to $82 \mathrm{mr}$. In this analysis the SAT system was used to tag two photon events by detecting electrons scattered close to the beam axis. 


\section{II.2 DATA ACQUISITION}

At SPEAR, the MARK II gathered data both at fixed center of mass energies and over energy scans. The SPEAR $J / \psi$ data set consists of $e^{+} e^{-}$annihilation events at the $J / \psi\left(E_{c m}=3.095 \mathrm{GeV}\right)$, while the SPEAR $q \bar{q}$ data is made up of three smaller data samples of 4.1 $\mathrm{GeV}$ to $6.7 \mathrm{GeV}$ representing the continuum above the $J / \psi$. During its operation at PEP, the MARK II collected a total of $220 p b^{-1}$ of data at $E_{c m}$ of $29 \mathrm{GeV}$. Approximately half of the data was collected with the drift chamber operating at reduced voltage, degrading the tracking efficiency. The PEP $q \bar{q}$ data set, consisting of $e^{+} e^{-}$annihilation events at $E_{c m}=29 \mathrm{GeV}$, is taken from the high quality half of the data. The PEP $\gamma \gamma$ data, made up of SAT-tagged two photon events, is drawn from the entire $220 \mathrm{pb}^{-1}$ collected at PEP. The energy of the tagging electron was required to be greater then $6 \mathrm{GeV}$. The data sets are summarized in Table 1.

\section{II.3 TRACK AND EVENT SELECTION}

In general, the cuts used here are fairly loose and were chosen to retain maximum statistics while selecting well measured hadronic events and minimizing contamination from backgrounds such as cosmic rays, beam-gas events, and lepton pair production processes.

Event selection begins with a search for charged tracks which have well measured momenta and project close to the primary vertex. The momentum of each accepted track is required to satisfy $p_{x y} \geq 0.1 \mathrm{GeV} / \mathrm{c}$, where $p_{x y}$ is the momentum component perpendicular to the beam axis. The distance of closest approach of the track to the beam axis is required to be $r_{z} \leq 5.0 \mathrm{~cm}$ along the beam axis and

$$
r_{x y} \leq \begin{cases}1.0 \mathrm{~cm} & \text { for } p_{x y}>1 \mathrm{GeV} / \mathrm{c} \\ \frac{1.0 \mathrm{~cm} \mathrm{GeV} / \mathrm{c}}{p_{x y}} & \text { for } p_{x y} \leq 1 \mathrm{GeV} / \mathrm{c}\end{cases}
$$

perpendicular to the beam axis. This cut reduces the fraction of tracks coming from relatively distant decay vertices, such as the $K^{0}$ and $\Lambda$. To ensure that the track is 
well tracked by the drift chamber, the angle between the track and the beam axis at the primary vertex must satisfy $|\cos \theta| \leq 0.79$.

Calorimeter tracks due to neutral particles are subjected to minimal quality cuts since they are only used in cluster finding and in calculating the total visible energy. Such a neutral track is used if it deposited an energy of $200 \mathrm{MeV}$ or more in the liquid-argon calorimeter, and if the shower center is $7 \mathrm{~cm}$ or more away from the closest projected drift chamber track.

Several cuts are made on the events defined by these charged and neutral tracks. The reconstructed primary vertex must be within $1 \mathrm{~cm}$ of the interaction point in the $x y$ plane and within $5.0 \mathrm{~cm}$ in the direction along the beam axis. The event must have three or more charged tracks. Contamination by simple QED events is reduced by two additional cuts. First, an event is rejected if it has three or four good charged tracks and contains at least one well identified $e$ or $\mu$. Second, in order to remove tau pairs, an event is rejected if it is determined to have two jets ${ }^{29}$ each of which has an effective mass less than $2.5 \mathrm{GeV}$. In order to remove two photon events from the sample of $e^{+} e^{-}$annihilation events, we require that the total visible energy $E_{\text {total }}=E_{\text {charged }}+E_{\text {neutral }}$ be greater than $0.25 E_{c m}$.

All particles which are not well identified electrons, muons, kaons, or protons are given pion masses. Well identified is defined for each particle as follows:

(1) a particle with $p \geq 1 \mathrm{GeV} / \mathrm{c}$ is called an electron if it satisfies the MARK II utility cuts ${ }^{30}$ for defining electrons, based on liquid-argon energy deposition and shower geometry;

(2) a particle with $p \geq 2 \mathrm{GeV} / \mathrm{c}$ is called a muon if it penetrates all four layers of the muon system;

(3) a particle with $p \leq 1 \mathrm{GeV} / c(p \leq 2 \mathrm{GeV} / \mathrm{c})$ is called a kaon (proton) if the TOF weight ${ }^{31}$ for a kaon (proton) is $W_{K}\left(W_{P}\right) \geq 0.35$, and if the measured time of flight is within $4 \sigma$ of the expected time for a kaon (proton) with momentum $p$.

We use Monte Carlo simulation to estimate and correct for the remaining fraction 
Table 1. A summary of the four data sets studied in this analysis.

\begin{tabular}{|c|c|c|c|}
\hline Data Set Name & Process & $E_{c m}(G e V)$ & $\mathcal{L}\left(p b^{-1}\right)$ \\
\hline SPEAR $J / \phi$ & $\psi \rightarrow$ hadrons & 3.1 & 0.4 \\
\hline PEP $\gamma \gamma$ & $\gamma \gamma \rightarrow$ hadrons & 29 & 198.0 \\
\hline SPEAR $q \Phi$ & $q q \rightarrow$ hadrons & $4.1-6.7$ & 13.2 \\
\hline PEP $q \Phi$ & $q \Phi \rightarrow$ hadrons & 29 & 113.2 \\
\hline
\end{tabular}

Table 2. Number of events on data summary tapes before any cuts, the number of events surviving event selection cuts, and the number of events contributing pairs and triplets.

\begin{tabular}{|c|c|c|c|c|}
\hline Data Set & $\begin{array}{l}\text { Number of } \\
\text { Events } \\
\text { Before Any Cuts }\end{array}$ & $\begin{array}{l}\text { Number of } \\
\text { Hadronic Events }\end{array}$ & $\begin{array}{l}\text { Number of } \\
\text { Analysis Events } \\
\text { (Pairs) }\end{array}$ & $\begin{array}{l}\text { Number of } \\
\text { Analysis Events } \\
\text { (Triplets) }\end{array}$ \\
\hline SPEAR $J / \psi$ & $1,285,000$ & 477,000 & 308,000 & 48,000 \\
\hline PEP $\gamma \tau$ & 264,000 & 42,000 & 28,000 & 6,000 \\
\hline SPEAR $q q$ & 495,000 & 78,000 & 51,000 & 16,000 \\
\hline PEP $q q$ & 182,000 & 54,000 & 52,000 & 45,000 \\
\hline
\end{tabular}

Table 3. The total number of pairs and triplets in each data set.

\begin{tabular}{|c|c|c|c|c|}
\hline Data Set & $N_{\pi^{ \pm} \pi^{ \pm}}$ & $N_{\pi^{ \pm} \pi}$ & $N_{\pi^{ \pm} \pi^{ \pm}}$ & $N_{\pi^{ \pm} \pi^{ \pm}}$ \\
\hline SPEAR $J / \psi$ & 484,000 & 813,000 & 55,000 & 436,000 \\
\hline PEP $\gamma \gamma$ & 61,000 & 94,000 & 13,000 & 73,000 \\
\hline SPEAR $q q$ & 149,000 & 224,000 & 36,000 & 216,000 \\
\hline PEP $q \Phi$ & 278,000 & 323,000 & 341,000 & $1,375,000$ \\
\hline
\end{tabular}


of particles misidentified as pions.

In order to eliminate photon conversion products which can be misidentified as an unlike charged pion pair, we apply a cut on the pair angle: $\cos \theta_{\text {pair }} \leq 0.999$. The same cut is applied to like charged pairs to preserve the phase space match between like and unlike pairs. This cut falls well within the first bin of our $Q^{2}$ distribution and does not measurably influence the fitted values of the parameters of interest.

Finally, a pion multiplicity cut is made on the event. Events with two pions favor unlike charged pairs. We therefore require that at least three pions and both charges be present for pair analysis. For similar reasons, a minimum multiplicity of five pions and both charges are required for triplet analysis.

The total number of events passing these cuts, together with the number of events on data summary tapes before the cuts, are listed for each data set in Table 2. Table 3 shows the total number of pions, pairs, and triplets in each data set. Note that not all events used to make pairs $\left(N_{\pi^{ \pm}} \geq 3\right)$ can be used to make triplets $\left(N_{\pi \pm} \geq 5\right)$. 


\section{STUDY AND REFERENCE SAMPLES}

In this analysis we examine the Bose-Einstein correlation in two study samples: like charged pion pairs and like-charged pion triplets. Unless otherwise noted, all of the comments apply to both study samples. We measure the Bose-Einstein enhancement by comparing the study sample to a reference sample. An ideal reference sample is a copy of the study sample in the absence of the Bose-Einstein correlation. In reality, reference samples available to us are only approximations of the ideal.

Momentum conservation and pion multiplicity together determine the kinematical phase space distribution of pion pairs and triplets independently of their charge combinations. Unlike charged pion pairs are therefore an obvious choice as a reference sample for the pair study sample, although resonances in the phase space of unlike pairs must be taken into account when studying the ratio of like to unlike pairs. An analysis of the triplet study sample is complicated by the need for a reference sample which is entirely free of the Bose-Einstein correlation. Any triplet of positive and negative pions $( \pm \pm \mp)$ contains one like charged pair and the corresponding pair Bose-Einstein correlation.

Combining pions from different events, or event mixing, is a well established method of approximating the ideal reference sample ${ }^{7}$. In this analysis we use an event mixing algorithm to create mixed cluster reference samples for pairs and triplets. In pair analysis, the mixed cluster reference sample is free of resonances such as the $K^{0}$ and $\rho^{0}$ that distort the phase space of the unlike charged pairs. In triplet analysis, the mixed cluster reference sample is entirely free of the BoseEinstein correlation, unlike the $\pm \pm \mp$ reference sample. The construction of the mixed cluster reference samples is described in appendix $\mathrm{C}$.

In Fig. 1, we show the $Q^{2}$ distributions of like and unlike pairs superimposed with the number of unlike pairs normalized to the number of like pairs in the $Q^{2}$ region 0.68 to $1.0 \mathrm{GeV}^{2}$. We can note the agreement in the phase space envelopes of the two samples, the Bose-Einstein enhancement in the region of small $Q^{2}$ in the like charged pairs, and the $K^{0}$ and $\rho^{0}$ signals in the unlike charged pairs. Figure 2 shows a similar comparison for like charged triplets and $\pm \pm \mp$ triplets in the variable $Q_{3 \pi}^{2}$. 


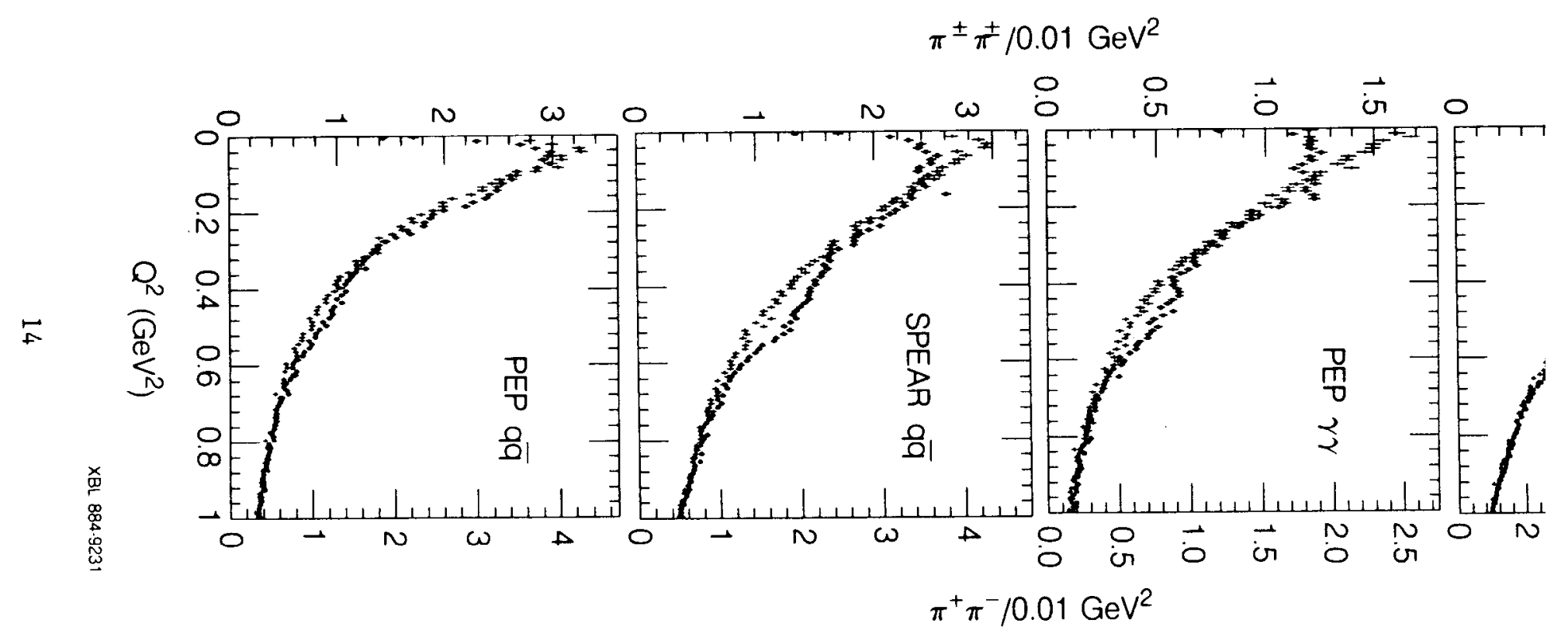

Fig. 1. The $Q^{2}$ distributions for like (crosses) and unlike (circles) charged pairs. two distributions are normalized to each other by rescaling the vertical of the unlike pair distribution so that the like and unlike distributions ov each other in the region of $0.20 \mathrm{GeV}^{2}<Q^{2}<0.36 \mathrm{GeV}^{2}$. Note the e of like charged pairs (Bose-Einstein enhancement) in the region of sma and the $K^{0}$ and $\rho^{0}$ resonance signals in the unlike charged pairs. 


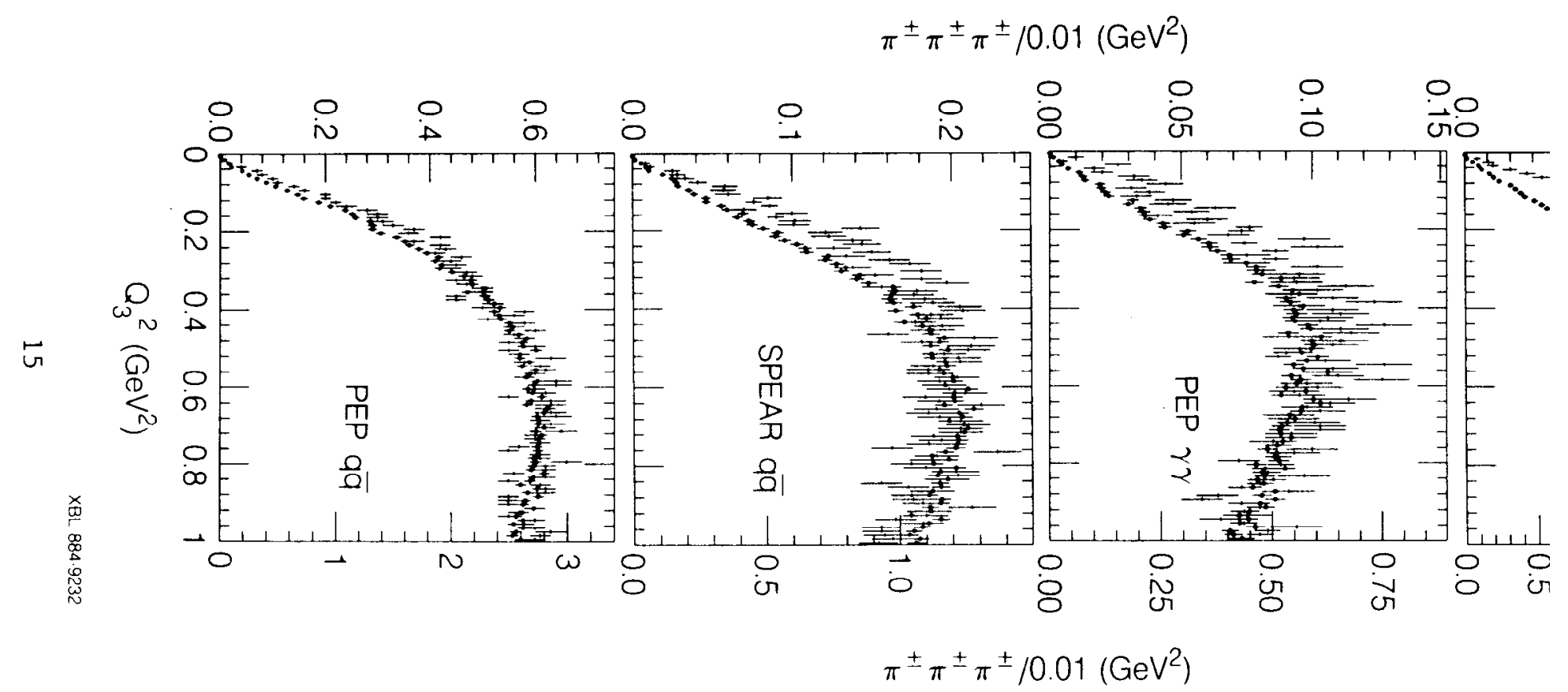

Fig. 2. The $Q_{3 \pi}^{2}$ distributions for like (crosses) and $\pm \pm \mp$ (circles) triplets. $T$. distributions are normalized to each other by rescaling the vertical : the $\pm \pm \mp$ triplet distribution so that the like and $\pm \pm \mp$ distributions $\mathrm{c}$ each other in the region of $0.50 \mathrm{GeV}^{2}<Q^{2}<1.00 \mathrm{GeV}^{2}$. Note the of like charged triplets (Bose-Einstein enhancement) in the region of $Q_{3 \pi}^{2}$. 
Figure 3 shows the uncorrected ratio of like to unlike charged pair distributions, while Figure 4 shows the uncorrected ratio of like to mixed cluster pair distributions. Figure 5 and Fig. 6 show the uncorrected ratios of like to $\pm \pm \mp$ and like to mixed cluster triplet distributions, respectively. 


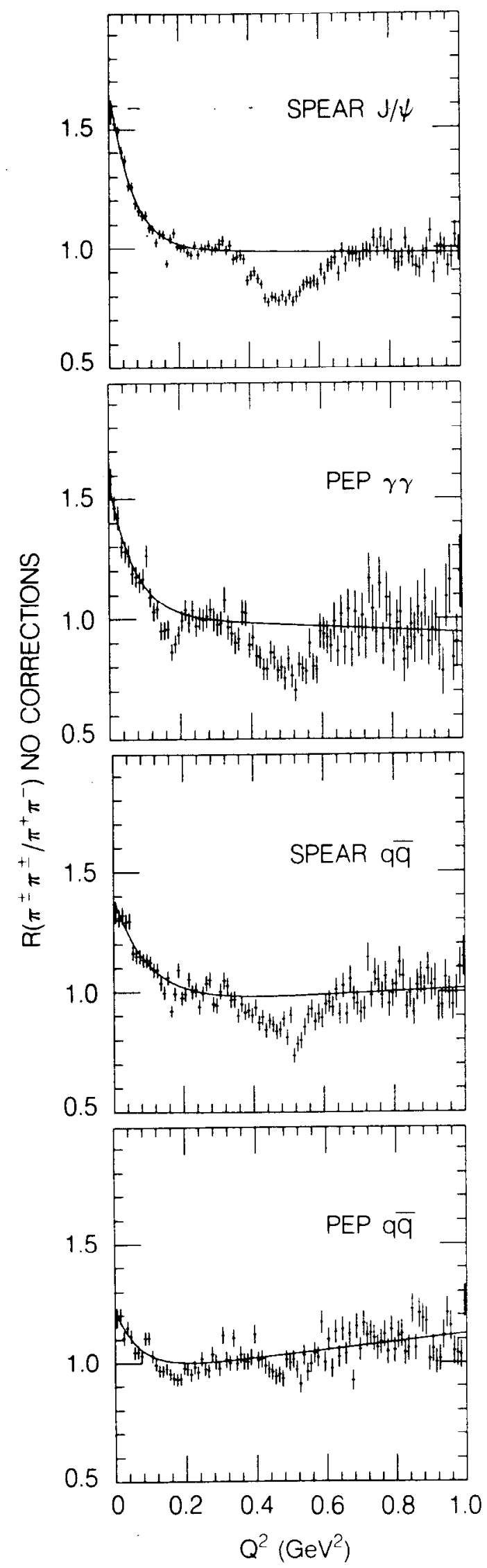

Fig. 3. Ratio of like to unlike charged pair distributions with no corrections applied. 


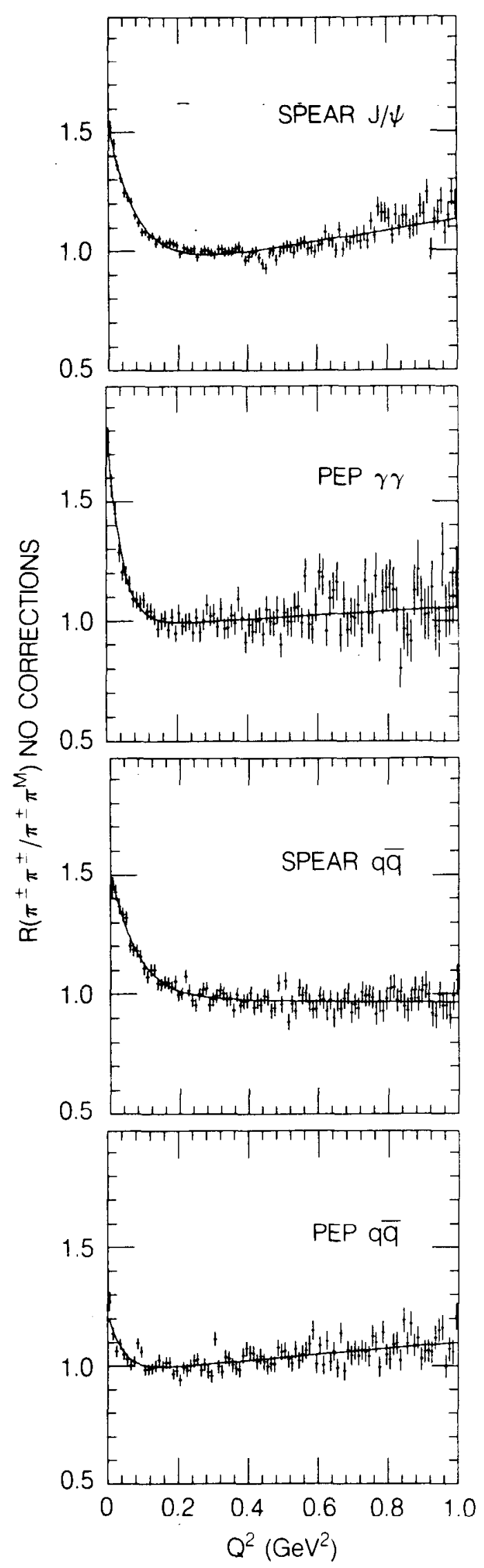

Fig. 4. Ratio of like to mixed cluster pair distributions with no corrections applied. 


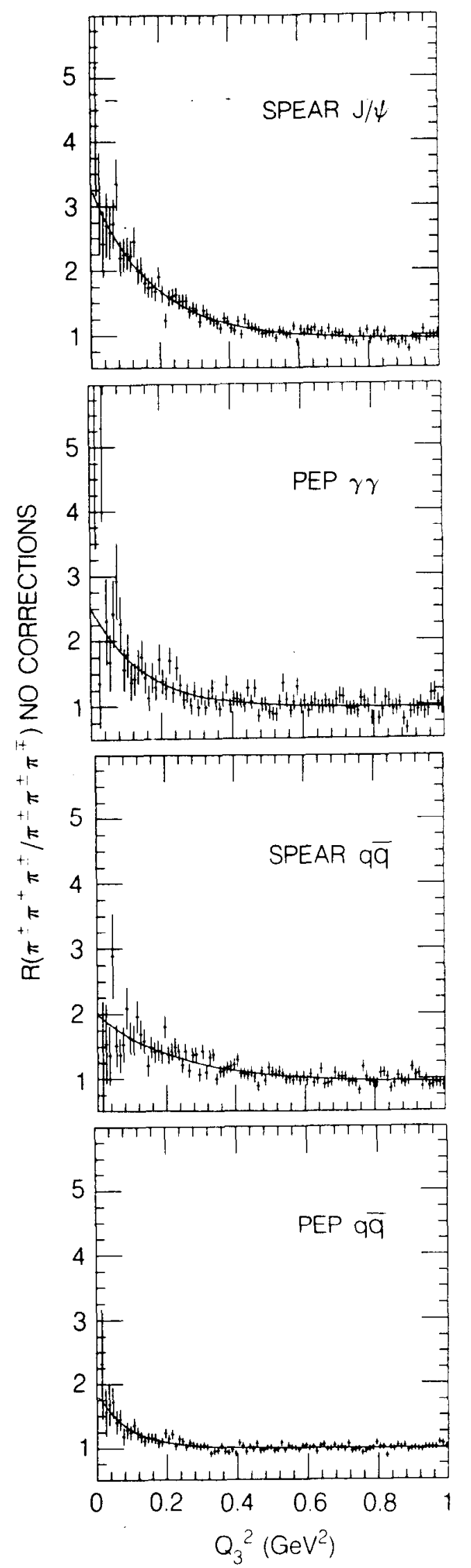

Fig. 5. Ratio of like to $\pm \pm \mp$ triplet distributions with no corrections applied. 


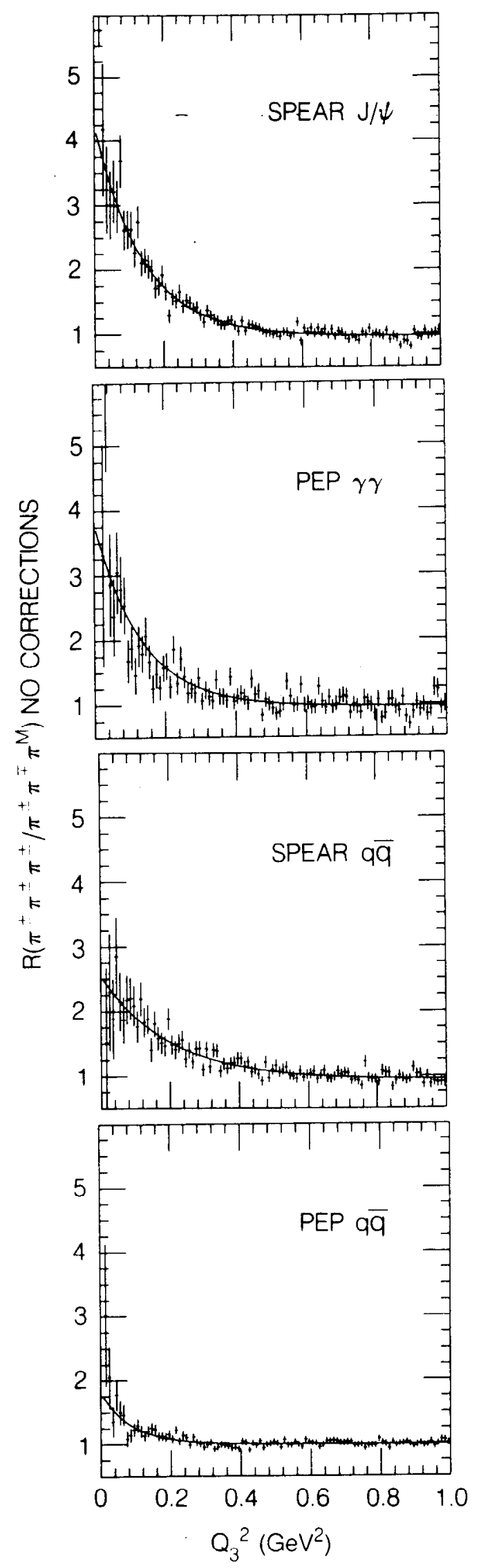

Fig. 6. Ratio of like to mixed cluster triplet distributions with no corrections applied. 


\section{CORRECTIONS}

Before we can study the Bose-Einstein correlation, we must understand what other effects are present in our study and reference samples. These can be divided into two general categories: other correlations arising from the hadronization process and detector efficiency effects.

\section{IV.1 FinAL STATE INTERACTIONS}

The final state of a charged pion pair is affected by the strong interaction and by the Coulomb force.

An estimate of the effects of the strong interaction on the measurement of the Bose-Einstein enhancement has been made by Suzuki ${ }^{32,33}$. The two like sign pions, which at low relative momentum are in an s-wave $I=2$ state, experience a repulsive final state interaction. Suzuki estimates the effect on $\lambda$ to be roughly a $15 \%$ reduction. Bowler ${ }^{33}$ estimates even a considerably larger reduction, but goes on to point out that for this to occur the pions would have to be at a range small compared to $1 \mathrm{fm}$. He argues that for an extended source, such as represented by a color string, only a small fraction of the pions are within the range of final state dipion interactions. In view of the uncertainties involved we make no attempt to correct for strong interaction effects. As we will show below, within the framework of Bose-Einstein correlations, there is no room for such corrections, in two of our data samples, without $\lambda$ significantly exeeding unity.

Two like (unlike) charged pions experience Coulomb repulsion (attraction), producing fewer (extra) pairs in the region of small relative momenta. The correction factor for this effect follows from the generalized Gamow factor, which is derived, for example, by Davydov ${ }^{34}$. The ratio of the corrected like to corrected unlike pairs then represents $R$ in the absence of the Coulomb interaction. Details of the Coulomb correction procedure in pair and triplet analyses are described in appendix $\mathrm{A}$. The net effect of the Coulomb correction is to increase the fitted value of $\lambda$ by about $11-13 \%$ over the uncorrected value in all four data sets. The value of $r$ is unaffected, within error, by this correction. Similarly in triplets, the fitted 
value of $\lambda_{3}$ is increased by $\approx 10 \%$.

\section{IV.2 PION MISIDENTIFICATION}

If one or both particles in a like charged pair are not really pions, the pair will not exhibit the Bose-Einstein correlation and will thus reduce the observed Bose-Einstein enhancement. Although the probability of pion misidentification is a function of particle momentum, the fraction of like charged pairs $T_{\pi \pi}$ in which both pions are correctly identified is found to be nearly independent of $Q^{2}$. This fraction and the reference sample distribution are used to calculate a pion misidentification correction for like charged pion pair and triplet distributions. Details of the pion misidentification correction are given in appendix $B$. The net effect of the pion misidentification correction is to increase the fitted value of $\lambda$ by about 20 to $25 \%$, depending on the data set. The value of $r$ is the same, within error, before and after this correction. In triplets, the pion misidentification correction raises the fitted value of $\lambda_{3}$ by about $30-38 \%$, again depending on the data set.

\section{IV.3 DRIFT CHAMBER PERFORMANCE}

The two track resolution limit of the drift chamber dictates how small a $Q^{2}$ and thus how large a parameter $r$ can be resolved. From a study of the difference between $\sqrt{Q_{\text {gen }}^{2}}$ calculated using Monte Carlo generated momenta and $\sqrt{Q_{\text {det }}^{2}}$ calculated using the reconstructed Monte Carlo tracks, we find a distribution that has a full width at half maximum of $\triangle Q=0.028 \mathrm{GeV}$ for pairs and $\triangle Q_{3}=0.05 \mathrm{GeV}$ for triplets. We thus estimate the maximum parameters $r$ and $r_{3}$ that can be observed in this experiment as

$$
r_{\max }=\frac{\hbar c}{\triangle Q} \approx 7 \mathrm{fm}
$$

and

$$
r_{3 \max }=\frac{\hbar c}{\Delta Q_{3}} \approx 4 \mathrm{fm}
$$

These are well outside the range of $\approx 1 \mathrm{fm}$, which is the typical value found in $e^{+} e^{-}$collision experiments. 
One of the major systematic errors in this analysis is the broadening of the Bose-Einstein enhancement due to the finite momentum resolution. To study this effect, we simulate the Bose-Einstein enhancement in Monte Carlo data by weighting each like charged pion pair by $R\left(Q^{2}\right)$ with $\lambda=1.00$ and $r=1.00 \mathrm{fm}$. Distributions in $Q_{g e n}^{2}$ and $Q_{d e t}^{2}$ are made for weighted like and non-weighted like pion pairs. For both $Q_{g e n}^{2}$ and $Q_{\text {det }}^{2}$ we form the ratio of weighted like to non-weighted like pairs and fit it to the expression for $R\left(Q^{2}\right)$. Comparing the fitted values of $\lambda$ and $r$ for $R\left(Q_{g e n}^{2}\right)$ with the fitted values for $R\left(Q_{d e t}^{2}\right)$, we assign a systematic error of $8 \%$ in $\lambda$ and $5 \%$ in $r$ due to the momentum resolution. An analogous procedure with triplets leads us to assign a systematic error of $15 \%$ in $\lambda_{3 \pi}$ and $5 \%$ in $r_{3 \pi}$.

Finally, we study the asymmetry in the detection of like versus unlike charged pairs with small pair angles by considering the distribution of pair angles in Monte Carlo data. In order to remove conversion pairs, where the $e^{+} e^{-}$are misidentified as a pion pair, we eliminate pairs with $\cos \theta>0.999(\theta<2.5$ degrees $)$ from both the like and unlike charged pair samples. A study of Monte Carlo simulated events shows that above $\approx \mathbf{2} .5$ degrees like and unlike pair angle distributions have the same shape, and hence the detection asymmetry is negligible.

\section{IV.4 OTHER CORRELATIONS}

The Bose-Einstein correlation modifies the phase space density of single pions, which in turn modifies the phase space of both the unlike charged pion pairs and the mixed cluster reference sample. The magnitude of this effect, also known as the residual correlation, is estimated using an iterative procedure ${ }^{35}$. We find that the magnitude of the residual correlation is a small fraction of the error in the fit to the parameters of interest and therefore negligible.

In the ratio of like to unlike pairs, shown in Fig. 3, there is evidence of long range momentum correlations in the form of a slow rise in $R$ with $Q^{2}$. We take this effect into account by modifying the expression for $R$ to include a factor $\left(1+\delta Q^{2}\right)$, where $\delta$ parameterizes the average rise with $Q^{2}$. The full expression used in fitting 
is then

$$
R=N\left(1+\delta Q^{2}\right)\left(1+\lambda e^{-r^{2} Q^{2}}\right),
$$

where $N$ is a normalization factor. 


\section{RESULTS AND DISCUSSION}

\section{V.1 Calculation and Fitting of the CoRrelation Function}

Before the ratio $R$ is made, the data are fully corrected by applying the Coulomb correction to both the study and unlike pion reference sample distributions and the pion misidentification correction to the study sample distributions. All the fits are done by minimizing the $\chi^{2}$ with the utility minimization routine MINUIT $^{36}$. The fit to the expression for $R\left(Q^{2}\right)$ (Eq. (1)) is done over

$$
Q^{2}\left(G e V^{2}\right)=[0.00-0.12,0.20-0.36,0.68-1.00]
$$

These regions avoid the $K^{0}$ and $\rho^{0}$ resonances in the unlike charged pairs. For consistency, the same regions are used in fitting the ratio of like to mixed cluster pairs. The fit parameters are $\lambda, r, \delta$, and $N$. The number of study and reference sample pairs are normalized to each other over the region $0.20 \mathrm{GeV}^{2}<Q^{2}<$ $0.36 \mathrm{GeV}^{2}$, but $N$ is retained in the fit to take care of small deviations from 1.00.

In analogy with the pair analysis, the fully corrected pion triplet ratio $R_{3}\left(Q_{3}^{2}\right)$ is fitted to

$$
R_{3}=N_{3}\left(1+\lambda_{3} e^{-r_{3}^{2} Q_{3}^{2}}\right)
$$

The fit to $R_{3}$ is done over

$$
Q_{3}^{2}\left(G e V^{2}\right)=[0.01-1.00]
$$

both for the $\pm \pm \mp$ reference sample and for the mixed cluster reference sample.

\section{V.2 Results of Pair ANalyses}

Pair analysis results are given in Table 4. The ratio of fully corrected like to unlike charged pair distributions are shown in Fig. 7, while the ratio of fully corrected like to mixed cluster pair distributions are shown in Fig. 8.

In the ratio of like to unlike pairs, we find that after all corrections are made the values of $\lambda$ for the SPEAR $J / \psi$ and PEP $\gamma \gamma$ data sets are close to the maximum 
Table 4.

Results of the fit to $R\left(Q^{2}\right)$ for the pion pair study sample with no corrections and with both Coulomb and pion misidentification corrections (fully corrected). The statistical error is shown first, followed by the systematic error.

\begin{tabular}{|c|c|c|c|c|}
\hline parameter & $\operatorname{SPEAR} J / \psi$ & PEP $\gamma \gamma$ & SPEAR $q q$ & PEP $q \bar{q}$ \\
\hline \multicolumn{5}{|c|}{ REFERENCE SAMPLE: UNLIKE CHARGED PAIRS } \\
\hline \multicolumn{5}{|c|}{ NO CORRECTIONS } \\
\hline$\lambda$ & $0.69 \pm 0.03 \pm 0.06$ & $0.56 \pm 0.05 \pm 0.05$ & $0.46 \pm 0.04 \pm 0.05$ & $0.28 \pm 0.02 \pm 0.02$ \\
\hline$r(f m)$ & $0.77 \pm 0.02 \pm 0.04$ & $0.74 \pm 0.07 \pm 0.04$ & $0.63 \pm 0.06 \pm 0.03$ & $0.75 \pm 0.03 \pm 0.04$ \\
\hline$\delta\left(\mathrm{GeV}^{-2}\right)$ & $-0.01 \pm 0.02 \pm 0.01$ & $-0.06 \pm 0.04 \pm 0.01$ & $0.07 \pm 0.04 \pm 0.01$ & $0.18 \pm 0.01 \pm 0.02$ \\
\hline $\boldsymbol{N}$ & $0.99 \pm 0.01 \pm 0.01$ & $1.00 \pm 0.03 \pm 0.01$ & $0.95 \pm 0.03 \pm 0.01$ & $0.95 \pm 0.01 \pm 0.01$ \\
\hline$x^{2} / \mathrm{DOF}$ & $57 / 53$ & $48 / 53$ & $65 / 53$ & $89 / 53$ \\
\hline \multicolumn{5}{|c|}{ REFERENCE SAMPLE: UNLIKE CHARGED PAIRS } \\
\hline \multicolumn{5}{|c|}{ FULLY CORRECTED } \\
\hline$\lambda$ & $1.00 \pm 0.03 \pm 0.08$ & $0.87 \pm 0.07 \pm 0.07$ & $0.66 \pm 0.04 \pm 0.05$ & $0.50 \pm 0.03 \pm 0.04$ \\
\hline$r(f m)$ & $0.81 \pm 0.02 \pm 0.05$ & $0.84 \pm 0.06 \pm 0.05$ & $0.71 \pm 0.04 \pm 0.04$ & $0.84 \pm 0.06 \pm 0.05$ \\
\hline$\delta\left(\mathrm{GeV}^{-2}\right)$ & $0.02 \pm 0.02 \pm 0.01$ & $-0.07 \pm 0.05 \pm 0.01$ & $0.03 \pm 0.04 \pm 0.01$ & $0.23 \pm 0.04 \pm 0.02$ \\
\hline$N$ & $0.98 \pm 0.01 \pm 0.01$ & $1.02 \pm 0.03 \pm 0.02$ & $0.97 \pm 0.02 \pm 0.01$ & $0.94 \pm 0.02 \pm 0.01$ \\
\hline$x^{2} / \mathrm{DOF}$ & $47 / 53$ & $50 / 53$ & $61 / 53$ & $94 / 53$ \\
\hline \multicolumn{5}{|c|}{ REFERENCE SAMPLE: MIXED CLUSTER PAIRS } \\
\hline \multicolumn{5}{|c|}{ NO CORRECTIONS } \\
\hline$\lambda$ & $0.70 \pm 0.02 \pm 0.06$ & $0.87 \pm 0.06 \pm 0.07$ & $0.55 \pm 0.03 \pm 0.05$ & $0.27 \pm 0.04 \pm 0.02$ \\
\hline$r(f m)$ & $0.77 \pm 0.02 \pm 0.04$ & $1.02 \pm 0.05 \pm 0.05$ & $0.75 \pm 0.04 \pm 0.04$ & $0.97 \pm 0.10 \pm 0.05$ \\
\hline$\delta\left(\mathrm{GeV}^{-2}\right)$ & $0.25 \pm 0.02 \pm 0.02$ & $0.06 \pm 0.04 \pm 0.01$ & $-0.02 \pm 0.02 \pm 0.01$ & $0.13 \pm 0.02 \pm 0.01$ \\
\hline$N$ & $0.95 \pm 0.01 \pm 0.01$ & $0.99 \pm 0.02 \pm 0.01$ & $0.99 \pm 0.01 \pm 0.01$ & $0.97 \pm 0.01 \pm 0.01$ \\
\hline$x^{2} / \mathrm{DOF}$ & $57 / 53$ & $50 / 53$ & $43 / 53$ & $91 / 53$ \\
\hline \multirow{2}{*}{\multicolumn{5}{|c|}{$\begin{array}{c}\text { REFERENCE SAMPLE: MIXED CLUSTER PAIRS } \\
\text { FULLY CORRECTED }\end{array}$}} \\
\hline & & & & \\
\hline$\lambda$ & $0.96 \pm 0.03 \pm 0.08$ & $1.20 \pm 0.08 \pm 0.10$ & $0.72 \pm 0.04 \pm 0.06$ & $0.45 \pm 0.03 \pm 0.04$ \\
\hline$r(f m)$ & $0.79 \pm 0.02 \pm 0.04$ & $1.05 \pm 0.05 \pm 0.06$ & $0.78 \pm 0.04 \pm 0.04$ & $1.01 \pm 0.09 \pm 0.06$ \\
\hline$\delta\left(\mathrm{GeV}^{-2}\right)$ & $0.31 \pm 0.03 \pm 0.02$ & $0.06 \pm 0.04 \pm 0.01$ & $-0.04 \pm 0.03 \pm 0.01$ & $0.18 \pm 0.03 \pm 0.01$ \\
\hline$N$ & $0.91 \pm 0.01 \pm 0.01$ & $0.99 \pm 0.02 \pm 0.01$ & $0.99 \pm 0.02 \pm 0.01$ & $0.96 \pm 0.02 \pm 0.01$ \\
\hline$x^{2} / \mathrm{DOF}$ & $62 / 53$ & $55 / 53$ & $45 / 53$ & $107 / 53$ \\
\hline
\end{tabular}




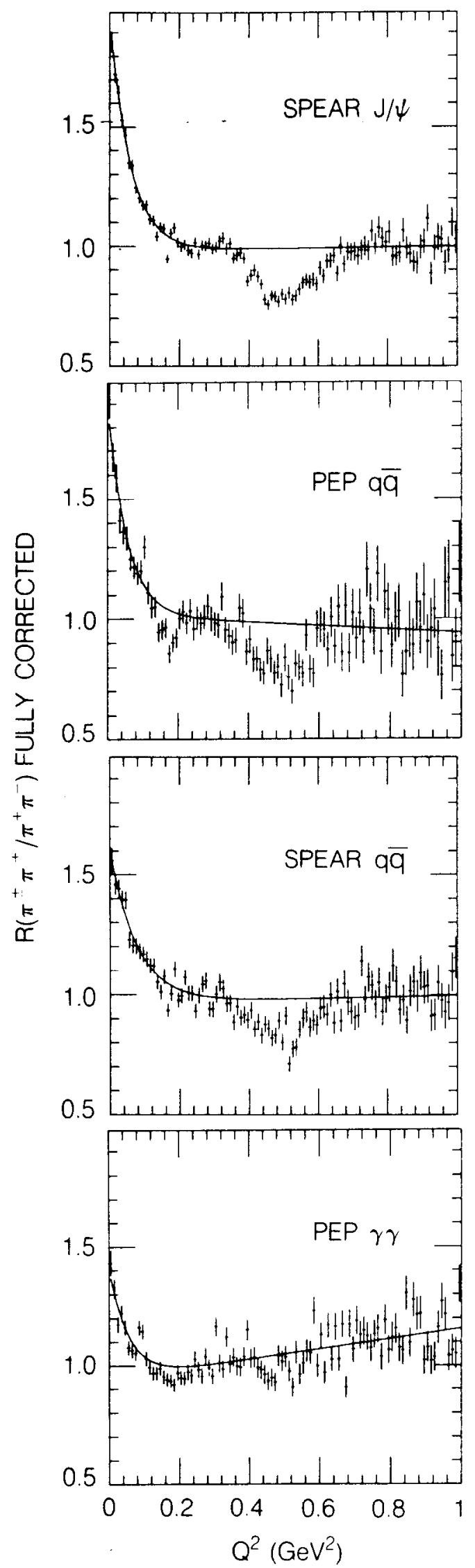

Fig. 7. Ratio of fully corrected like to unlike charged pair distributions and the fitted curves. 


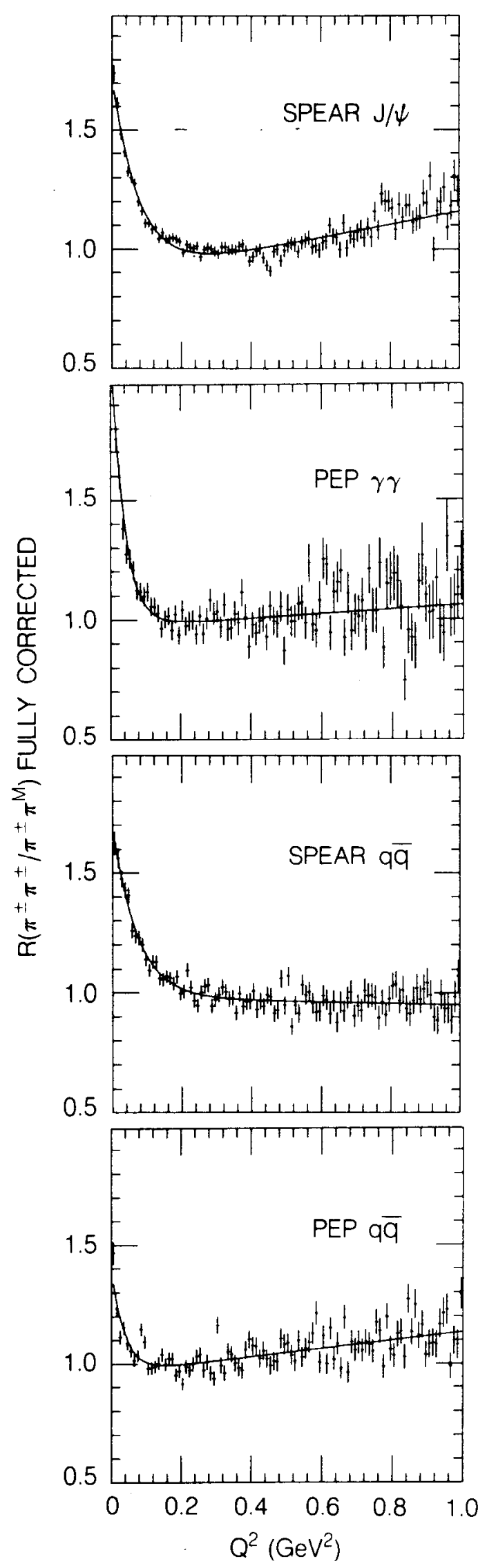

Fig. 8. Ratio of fully corrected like to mixed cluster pair distributions and the fitted curves. 
expected value of 1.00, while the $\lambda$ values for the SPEAR $q \bar{q}$ and PEP $q \bar{q}$ data sets are significantly smaller than the maximum. The value of $r$ is nearly independent of applied corrections and is fairly constant between $0.75 \mathrm{fm}$ to $0.85 \mathrm{fm}$ for all data sets.

Using the mixed cluster reference sample, we note that the $K^{0}$ and $\rho^{0}$ resonances clearly evident in Fig. 7 (ratio of like to unlike charged pairs) are absent as expected in Fig. 8 (ratio of like to mixed cluster pairs). Comparison of the parameter $\delta$ gives a measure of how well mixed cluster pairs reproduce the phase space distribution of unlike pairs at large $Q^{2}$. Values of $\delta$ for the two reference samples are the same within errors in the SPEAR $q \bar{q}$, PEP $\gamma \gamma$, and PEP $q \bar{q}$ data sets, while the agreement is slightly worse in the $\operatorname{SPEAR} J / \psi$ data. The small differences in $\lambda$ and $r$ found using the two reference samples represent a model dependent systematic error on the measured quantities. We consider the reasonable agreement between the two reference samples in pair analysis as a test of the mixed cluster reference sample prior to its use in triplet analysis.

\section{V.3 RESUltS OF TRIPLET ANALYSES}

Results of triplet analyses are shown in Table 5, which contains the results for both the ratio of like to $\pm \pm \mp$ triplets and the ratio of like to mixed cluster triplets. Figure 9 shows the ratio of fully corrected like to mixed cluster triplet distributions.

Since the $\pm \pm \mp$ reference sample contains one like charged pair, it is not surprising that $\lambda_{3}$ found with this reference sample is less than the maximum expected value of 5.00. However, the $\pm \pm \mp$ reference sample clearly shows that the SPEAR $J / \psi$ and PEP $\gamma \gamma$ data sets have significantly higher values of $\lambda_{3}$ than the other two data sets.

Using the mixed cluster triplet reference sample, we obtain values of $\lambda_{3}$ close to the maximum of 5.00 for the fully corrected SPEAR $J / \psi$ and PEP $\gamma \gamma$ data sets. Fits to the fully corrected SPEAR $q \bar{q}$ and PEP $q \bar{q}$ data sets show a half to a third of the maximum value of $\lambda_{3}$.

We find that the two reference samples give nearly the same values of $r_{3}$, and 
Table 5 .

Results of fits to $R_{3}\left(Q_{3}^{2}\right)$ for the pion triplet study sample with no corrections and with both Coulomb and pion misidentification corrections (fully corrected). The statistical error is shown first, followed by the systematic error.

\begin{tabular}{|c|c|c|c|c|}
\hline parameter & $\operatorname{SPEAR} J / \psi$ & PEP $\boldsymbol{\gamma} \gamma$ & SPEAR $q q$ & PEP $q \Phi$ \\
\hline \multicolumn{5}{|c|}{ REFERENCE SAMPLE: $\pm \pm \mp$ TRIPLETS } \\
\hline \multicolumn{5}{|c|}{ NO CORRECTIONS } \\
\hline$\lambda_{9}$ & $2.46 \pm 0.17 \pm 0.31$ & $1.61 \pm 0.42 \pm 0.20$ & $1.12 \pm 0.12 \pm 0.14$ & $0.86 \pm 0.17 \pm 0.11$ \\
\hline$r_{3}(f m)$ & $0.47 \pm 0.02 \pm 0.03$ & $0.51 \pm 0.05 \pm 0.03$ & $0.41 \pm 0.03 \pm 0.03$ & $0.66 \pm 0.04 \pm 0.04$ \\
\hline$N$ & $0.95 \pm 0.01 \pm 0.01$ & $0.97 \pm 0.02 \pm 0.01$ & $0.94 \pm 0.02 \pm 0.01$ & $0.99 \pm 0.01 \pm 0.01$ \\
\hline$\chi^{2} /$ DOF & $99 / 95$ & $112 / 95$ & $106 / 95$ & $86 / 95$ \\
\hline \multicolumn{5}{|c|}{ REFERENCE SAMPLE: $\pm \pm \mp$ TRIPLETS } \\
\hline \multicolumn{5}{|c|}{ FULLY CORRECTED } \\
\hline$\lambda_{3}$ & $3.83 \pm 0.24 \pm 0.48$ & $2.61 \pm 0.40 \pm 0.33$ & $1.83 \pm 0.18 \pm 0.23$ & $1.60 \pm 0.25 \pm 0.20$ \\
\hline$r_{3}(f m)$ & $0.48 \pm 0.01 \pm 0.03$ & $0.47 \pm 0.04 \pm 0.03$ & $0.42 \pm 0.03 \pm 0.03$ & $0.64 \pm 0.04 \pm 0.04$ \\
\hline$N$ & $0.93 \pm 0.02 \pm 0.01$ & $0.93 \pm 0.04 \pm 0.01$ & $0.92 \pm 0.03 \pm 0.01$ & $0.99 \pm 0.01 \pm 0.01$ \\
\hline$\chi^{2} / \mathrm{DOF}$ & $107 / 95$ & $123 / 95$ & $115 / 95$ & $94 / 95$ \\
\hline \multicolumn{5}{|c|}{ REFERENCE SAMPLE: MLXED CLUSTER TRIPLETS } \\
\hline \multicolumn{5}{|c|}{ NO CORRECTIONS } \\
\hline$\lambda_{3}$ & $3.37 \pm 0.24 \pm 0.41$ & $2.94 \pm 0.63 \pm 0.35$ & $1.69 \pm 0.16 \pm 0.22$ & $0.83 \pm 0.16 \pm 0.11$ \\
\hline$r_{3}(f m)$ & $0.53 \pm 0.01 \pm 0.03$ & $0.54 \pm 0.04 \pm 0.03$ & $0.44 \pm 0.02 \pm 0.03$ & $0.66 \pm 0.04 \pm 0.04$ \\
\hline $\boldsymbol{N}$ & $0.97 \pm 0.01 \pm 0.01$ & $0.97 \pm 0.02 \pm 0.01$ & $0.95 \pm 0.02 \pm 0.01$ & $0.99 \pm 0.01 \pm 0.01$ \\
\hline$\chi^{2} / \mathrm{DOF}$ & $92 / 95$ & $119 / 95$ & $94 / 95$ & $90 / 95$ \\
\hline \multicolumn{5}{|c|}{ REFERENCE SAMPLE: MDXED CLUSTER TRIPLETS } \\
\hline \multicolumn{5}{|c|}{ FULLY CORRECTED } \\
\hline$\lambda_{3}$ & $4.97 \pm 0.33 \pm 0.62$ & $4.56 \pm 0.45 \pm 0.57$ & $2.58 \pm 0.24 \pm 0.33$ & $1.54 \pm 0.23 \pm 0.19$ \\
\hline$r_{3}(f m)$ & $0.53 \pm 0.01 \pm 0.03$ & $0.55 \pm 0.01 \pm 0.03$ & $0.45 \pm 0.02 \pm 0.03$ & $0.64 \pm 0.04 \pm 0.04$ \\
\hline$N$ & $0.96 \pm 0.02 \pm 0.01$ & $0.96 \pm 0.02 \pm 0.01$ & $0.93 \pm 0.02 \pm 0.01$ & $0.99 \pm 0.01 \pm 0.01$ \\
\hline$\chi^{2} / \mathrm{DOF}$ & $100 / 95$ & $125 / 95$ & $101 / 95$ & $90 / 95$ \\
\hline
\end{tabular}




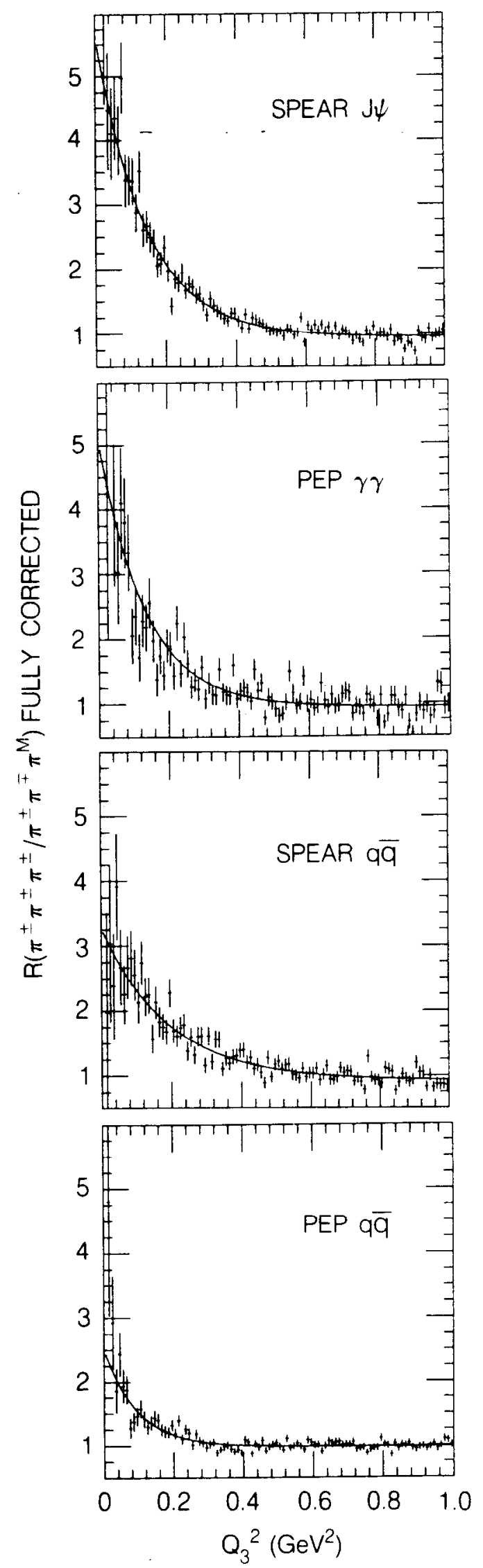

Fig. 9. Ratio of fully corrected like to mixed cluster triplet distributions and the fitted curves. 
that $r_{3}$ appears to be nearly uniform over the four data sets. Only the PEP $q \bar{q}$ data has a value of $r_{3}$ slightly higher than the other three data sets.

Using the mixed cluster reference samples and fully corrected data, we find a similar pattern in $\lambda$ and $\lambda_{3}$ : the SPEAR $J / \psi$ and PEP $\gamma \gamma$ data show both $\lambda$ and $\lambda_{3}$ values close to their theoretical maximum, while the SPEAR $q \bar{q}$ and PEP $q \bar{q}$ data show distinctly lower values (on the order of a half for $\lambda$ and a third for $\lambda_{3}$ ). Although triplet analysis is statistically weaker than pair analysis, it seems to be a more sensitive measure of the Bose-Einstein correlation than the latter. This is not surprising, since the Bose-Einstein correlation in triplets involves three correlated pairs plus a purely triplet correlation term ${ }^{37}$. Comparing $r_{3}$ in triplets with $\tau$ in pairs, we find

$$
r^{2} / 3<r_{3}^{2}<r^{2} / 2
$$

similar to results reported by other $e^{+} e^{-}$experiments ${ }^{9}$.

As a consistency check, we study the ratio of the $\pm \pm \mp$ triplets divided by the mixed cluster triplets (uncorrected reference sample distributions). This ratio is expected to exhibit a Bose-Einstein enhancement due to the one like charged pair present in the $\pm \pm \mp$ reference sample. We fit the expression for $R_{3}\left(Q_{3}^{2}\right)$ to the SPEAR $J / \psi$ data, the only data set large enough to give a statistically significant result, and find

$$
\lambda_{3}=0.53 \pm 0.15, \quad r_{3}=0.77 \pm 0.07 \mathrm{fm} .
$$

Although we can fit only the SPEAR $J / \psi$ data set, all four data sets show some enhancement near $Q_{3}^{2}=0$. This is consistent with the expectation that the mixed cluster reference sample behaves like a triplet of non-identical pions.

\section{V.4 Bose- Einstein Correlation in PEP $\gamma \gamma$ Pairs}

In the PEP $\gamma \gamma$ data, different hadronization mechanisms are expected to dominate in different regions of $p_{T}$, where $p_{T}$ is the component of each hadron's threemomentum perpendicular to the beam axis. We have studied PEP $\gamma \gamma$ data by characterizing events by their $p_{T \text { max }}$, the maximum pion $p_{T}$ in an event, relative 
to the beam axis, to find out how the observed Bose-Einstein correlation varies accross the transition between the two hadronization processes ${ }^{10}$. In the region of high $p_{T \text { max }}$ the two photons undergo hard scattering into $q \bar{q}$, while for low $p_{T \max }$ they annihilate predominantly in their vector meson form (VDM).

PEP $\gamma \gamma$ data is divided into three bins of $p_{T \text { max }}$, with roughly equal numbers of events. In each bin, the ratio of fully corrected like to unlike charged pair distributions is fitted to $R\left(Q^{2}\right)$, and the results are shown in Table 6. We see a clear decrease of $\lambda$ with $p_{T \max }$. The fully corrected ratio shows a nearly maximum value of $\lambda$ in the lowest $p_{T \max }$ bin, where hadronization proceeds through vector meson annihilation. In the highest $p_{T \max }$ bin, where hard scattering becomes increasingly important, $\lambda$ is significantly less than the maximum value. Within errors, the value of $r$ is nearly independent of $p_{T \max }$.

\section{V.5 BOSE-EINSTEIN CORRELATION IN COMPONENTS OF $Q^{2}$}

We first consider components of $Q^{2}$ with respect to the Kopylov axis defined in section I. Unlike the beam direction in nuclear collision experiments or the jet axis in two-jet events, the Kopylov axis can be defined pair by pair in any kind of pion production data. Two-dimensional histograms of like, unlike, and mixed cluster pairs are cumulated in three sets of variables: $\left(q_{T}^{2}, q_{L}^{2}\right),\left(q_{T}^{2}, q_{0}^{2}\right)$, and $\left(q_{T}^{2}, q_{L^{\prime}}^{2}\right)$. No corrections are made in this analysis for Coulomb effects or for pion misidentification. The results of the $Q^{2}$ analysis of pion pairs leads us to expect that the Coulomb and pion misidentification corrections would serve to increase the fitted value of $\lambda$, while the parameters $r_{T}, r_{L^{\prime}}, r_{L}$, and $r_{0}$ would remain as insensitive to these corrections as $r$ is. The ratios of like to reference sample pairs are fitted to the expressions for $R\left(q_{T}^{2}, q_{L}^{2}\right), R\left(q_{T}^{2}, q_{0}^{2}\right)$, and $R\left(q_{T}^{2}, q_{L^{\prime}}^{2}\right)$ given in section I. We average the fitted parameters for the ratios of like to unlike distributions and of like to mixed cluster distributions. In this way we reduce the effect of the $K^{0}$ and $\rho^{0}$ phase space distortions present in the unlike pairs. We take half of the difference between the two fitted values as part of the systematic error to the measured value. Results of these calculations are listed in Table 7, and some of the corresponding 
Table 6.

In each of three bins of $p_{T \text { max }}$, the ratio of fully corrected like to unlike charged pair distributions is fitted to $R\left(Q^{2}\right)$, and the results are shown here.

\begin{tabular}{|c|c|c|c|}
\hline$p_{T_{\max }}(\mathrm{GeV} / \mathrm{c})$ & $0.0-0.5$ & $0.5-0.7$ & $>0.7$ \\
\hline$N_{\pi^{ \pm} \pm} / N_{\pi+\pi-}$ & $19,480 / 30,911$ & $21,340 / 32,506$ & $20,922 / 31,243$ \\
\hline \multicolumn{4}{|c|}{ FULLY CORRECTED } \\
\hline$\lambda$ & $1.03 \pm 0.09 \pm 0.06$ & $0.84 \pm 0.08 \pm 0.05$ & $0.78 \pm 0.11 \pm 0.04$ \\
\hline$r(f m)$ & $0.76 \pm 0.07 \pm 0.03$ & $0.63 \pm 0.09 \pm 0.03$ & $0.83 \pm 0.08 \pm 0.03$ \\
\hline
\end{tabular}

Table 7.

Results of fits to two-dimensional distributions in Kopylov variables. No corrections have been applied to this data. The calculation of these values and the corresponding statistical (first) and systematic (second) errors is discussed in the text. Values of the parameters $r_{T}, r_{L^{\prime}}, r_{0}$, and $r_{L}$ are in $f m$. We give the $\chi^{2}$ for the unlike pair analysis first and for the mixed cluster analysis second.

\begin{tabular}{|c|c|c|c|}
\hline Data Set & $\left(q_{T}^{2}, q_{L^{\prime}}^{2}\right)$ & $\left(q_{T}^{2}, q_{0}^{2}\right)$ & $\left(q_{T}^{2}, q_{L}^{2}\right)$ \\
\hline \multirow{3}{*}{ SPEAR $J / \psi$} & $\lambda=0.74 \pm 0.02 \pm 0.09$ & $\lambda=0.70 \pm 0.02 \pm 0.06$ & $\lambda=0.77 \pm 0.02 \pm 0.07$ \\
& $r_{T}=0.77 \pm 0.02 \pm 0.09$ & $r_{T}=0.85 \pm 0.02 \pm 0.05$ & $r_{T}=0.84 \pm 0.02 \pm 0.04$ \\
& $r_{L^{\prime}}=0.88 \pm 0.05 \pm 0.07$ & $r_{0}=0.64 \pm 0.03 \pm 0.16$ & $r_{L}=0.58 \pm 0.02 \pm 0.08$ \\
& $x^{2} / D O F=1.90,1.26$ & $x^{2} / D O F=1.58,1.38$ & $x^{2} / D O F=1.59,1.24$ \\
\hline \multirow{5}{*}{ PEP $\gamma \gamma$} & $\lambda=0.93 \pm 0.10 \pm 0.11$ & $\lambda=0.84 \pm 0.05 \pm 0.12$ & $\lambda=0.89 \pm 0.07 \pm 0.10$ \\
& $r_{T}=0.86 \pm 0.06 \pm 0.20$ & $r_{T}=1.00 \pm 0.05 \pm 0.15$ & $r_{T}=0.97 \pm 0.06 \pm 0.16$ \\
& $r_{L^{\prime}}=0.93 \pm 0.13 \pm 0.07$ & $r_{0}=0.45 \pm 0.05 \pm 0.03$ & $r_{L}=0.46 \pm 0.05 \pm 0.03$ \\
& $x^{2} / D O F=1.21,1.02$ & $x^{2} / D O F=1.30,1.02$ & $x^{2} / D O F=1.09,1.06$ \\
\hline \multirow{5}{*}{ SPEAR $q q$} & $\lambda=0.58 \pm 0.03 \pm 0.07$ & $\lambda=0.54 \pm 0.03 \pm 0.08$ & $\lambda=0.57 \pm 0.03 \pm 0.06$ \\
& $r_{T}=0.66 \pm 0.04 \pm 0.08$ & $r_{T}=0.82 \pm 0.05 \pm 0.03$ & $r_{T}=0.78 \pm 0.05 \pm 0.06$ \\
& $r_{L^{\prime}}=0.73 \pm 0.07 \pm 0.06$ & $r_{0}=0.45 \pm 0.04 \pm 0.03$ & $r_{L}=0.39 \pm 0.04 \pm 0.03$ \\
& $x^{2} / D O F=1.13,1.16$ & $x^{2} / D O F=1.00,1.19$ & $x^{2} / D O F=1.09,1.18$ \\
\hline \multirow{3}{*}{ PEP $q q$} & $\lambda=0.40 \pm 0.20 \pm 0.09$ & $\lambda=0.25 \pm 0.06 \pm 0.03$ & $\lambda=0.34 \pm 0.11 \pm 0.04$ \\
& $r_{T}=0.90 \pm 0.12 \pm 0.04$ & $r_{T}=0.94 \pm 0.15 \pm 0.16$ & $r_{T}=1.06 \pm 0.15 \pm 0.25$ \\
& $r_{L^{\prime}}=1.41 \pm 0.45 \pm 0.50$ & $r_{0}=0.49 \pm 0.15 \pm 0.22$ & $r_{L}=0.64 \pm 0.18 \pm 0.25$ \\
& $x^{2} / D O F=1.13,1.03$ & $x^{2} / D O F=0.90,0.98$ & $x^{2} / D O F=1.13,1.15$ \\
\hline
\end{tabular}


two-dimensional distributions are shown in Fig. 10 and Fig. 11.

The values of $\lambda$ found by fitting these uncorrected two-dimensional distributions are systematically slightly higher than the values of $\lambda$ found by fitting uncorrected distributions in $Q^{2}$ (Table 4). This effect can also be seen by fitting slices of the (for example) $\left(q_{T}^{2}, q_{L^{\prime}}^{2}\right)$ distribution. These slices and their fitted curves are shown in Figure 12 for the SPEAR $J / \psi$ data. Excluding the $K^{0}$ and $\rho^{0}$ regions from the fit, we find (statistical errors only):

$$
\begin{aligned}
& \text { for }\left(q_{T}^{2}, \quad 0.00 \mathrm{GeV}^{2}<q_{L^{\prime}}^{2}<0.06 \mathrm{GeV}^{2}\right) \text {, } \\
& \lambda=0.65 \pm 0.02 \quad \text { and } \quad r_{T}=0.78 \pm 0.01 \mathrm{fm} \text {; } \\
& \text { for }\left(0.00 \mathrm{GeV}^{2}<q_{T}^{2}<0.06 \mathrm{GeV}^{2}, q_{L^{\prime}}^{2}\right) \text {, } \\
& \lambda=0.57 \pm 0.03 \quad \text { and } \quad r_{L^{\prime}}=0.76 \pm 0.06 \mathrm{fm} .
\end{aligned}
$$

These $\lambda$ 's are smaller than the $\lambda$ derived from the two-dimensional $\left(q_{T}^{2}, q_{L^{\prime}}^{2}\right)$ distribution, because the Bose-Einstein enhancement falls off significantly within the width of the slice. Averaging over the slice, therefore, reduces the fitted $\lambda$. In the same way, a one dimensional distribution in $Q^{2}$ averages over the enhancement peak in the components of $Q^{2}$, and the resulting fitted value of $\lambda$ is lower in $R\left(Q^{2}\right)$. Within error, the measured radii are the same in the two slices as in the $\left(q_{T}^{2}, q_{L^{\prime}}^{2}\right)$ distribution.

The value of $r_{0} \approx r_{L}<r_{T} \approx r_{L^{\prime}}$ in all four data sets, with $r_{T}$ and $r_{L^{\prime}}$ approximately equal to the radius $r$ found in the fits to $R\left(Q^{2}\right)$. Similar observations have been reported by $\mathrm{CLEO}^{8}$ and $\mathrm{TASSO}^{9}$.

We also study $r_{T}, r_{L^{\prime}}$, and $r_{L}$ defined with respect to the jet axis using a subset of PEP $q \bar{q}$ data consisting of two-jet events. Again, no corrections have been applied to the data. Following the procedure used in the Kopylov axis analysis, we calculate the values shown in Table 8 . The parameter $\lambda$ does not differ much for the three distributions, and these values of $\lambda$ are within errors of the values found 

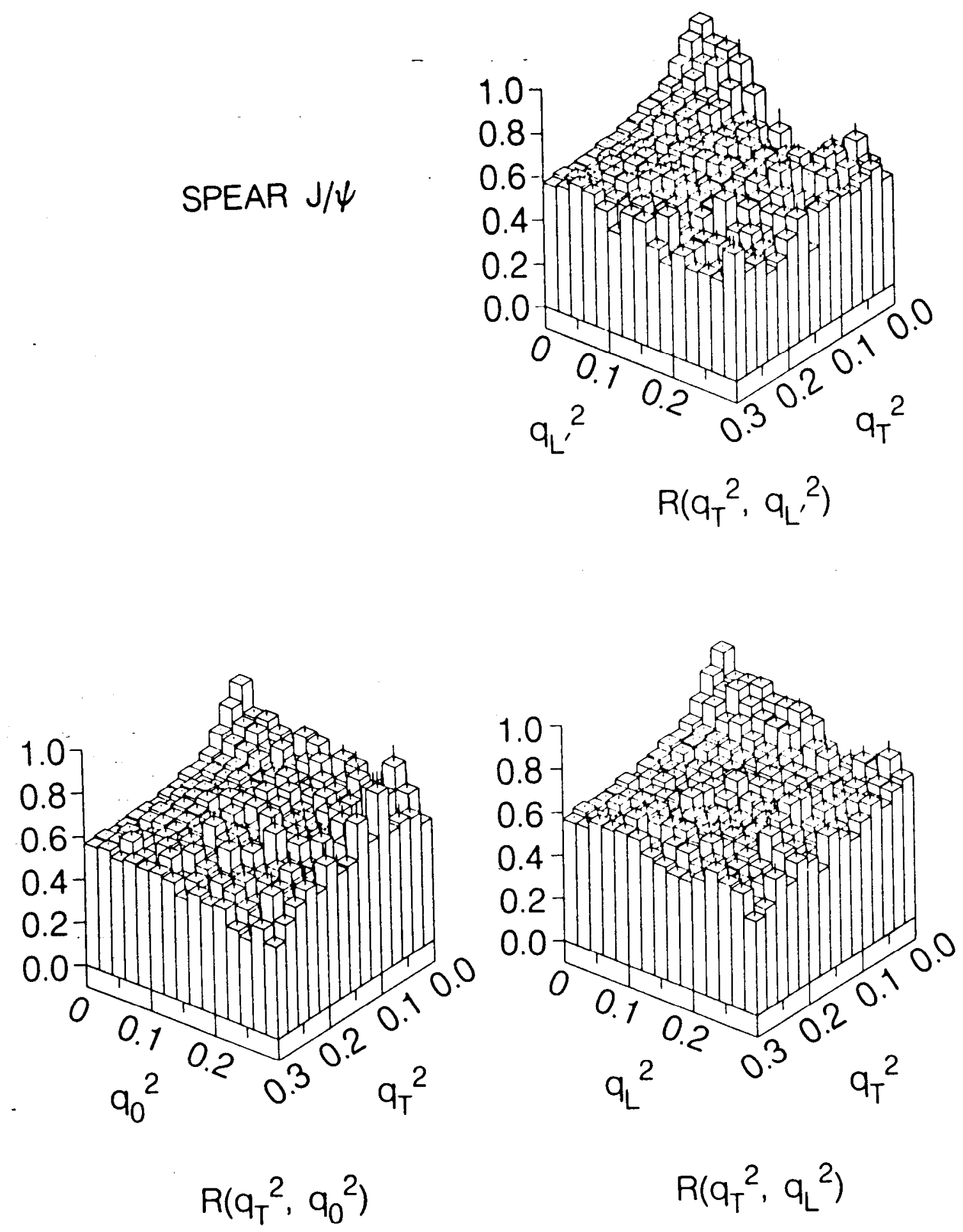

$$
R\left(q_{T}^{2}, q_{L}^{2}\right)
$$

Fig. 10. The ratio of like to unlike pairs (no corrections) in the SPEAR $J / \psi$ data. The variables $q_{T}^{2}, q_{L}^{2}$, and $q_{L^{\prime}}^{2}$ are defined with respect to the Kopylov axis, and all variables are given in $\mathrm{GeV}^{2}$. 


\section{$R\left(q_{T}{ }^{2}, q_{L^{\prime}}{ }^{2}\right)$}

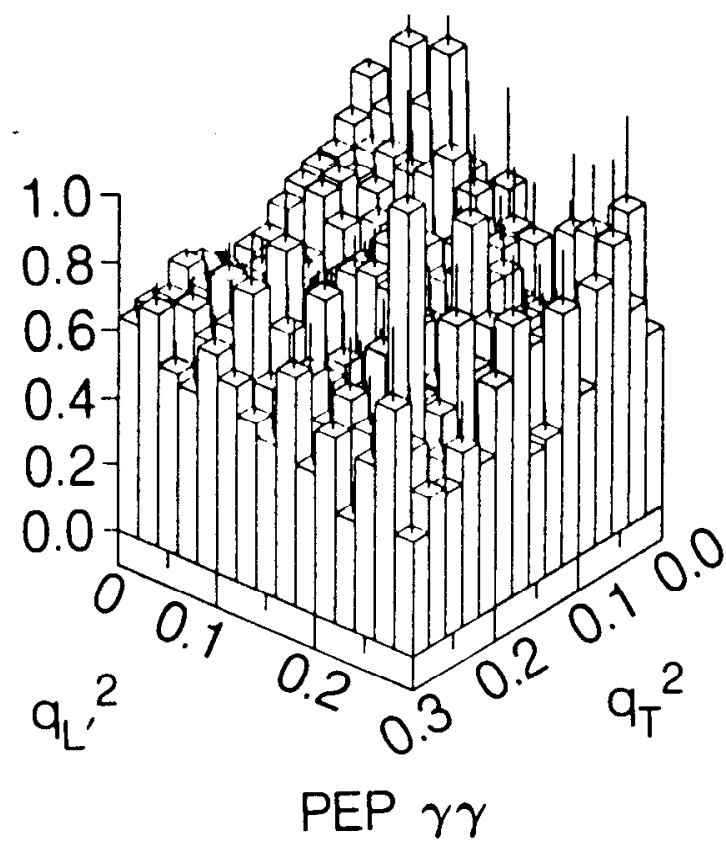

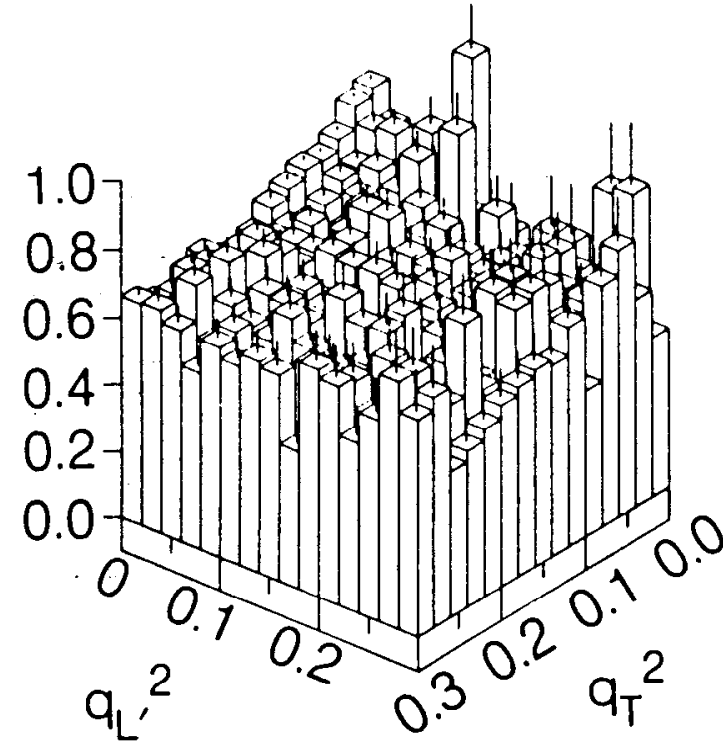

SPEAR $q \bar{q}$

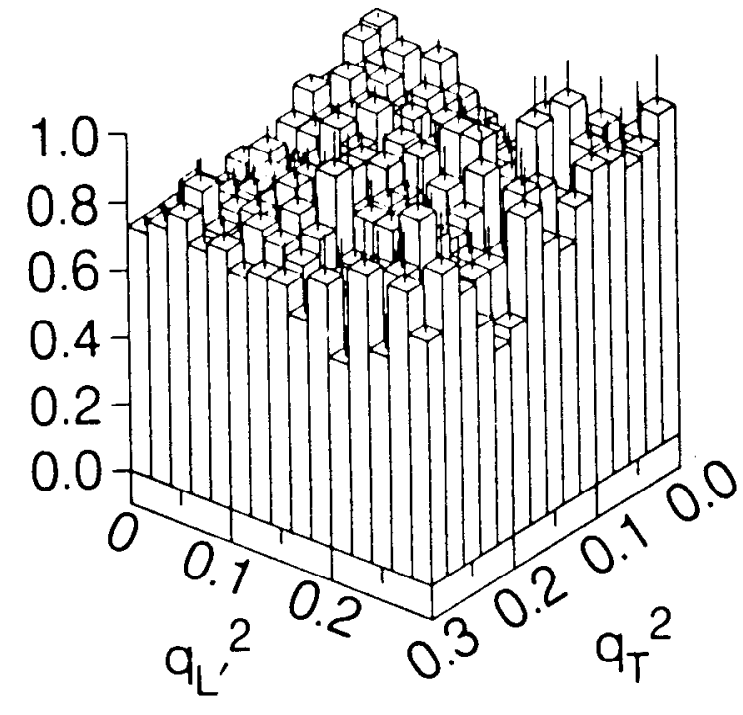

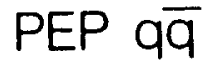

Fig. 11. The ratio of like to unlike pairs $R\left(q_{T}^{2}, q_{L^{\prime}}^{2}\right)$ (no corrections) in the SPEAR $q \bar{q}, \operatorname{PEP} \gamma \gamma$, and PEP $q \bar{q}$ data. The variables $q_{T}^{2}$ and $q_{L^{\prime}}^{2}$ are defined with respect to the Kopylov axis and are given in $\mathrm{GeV}^{2}$. 


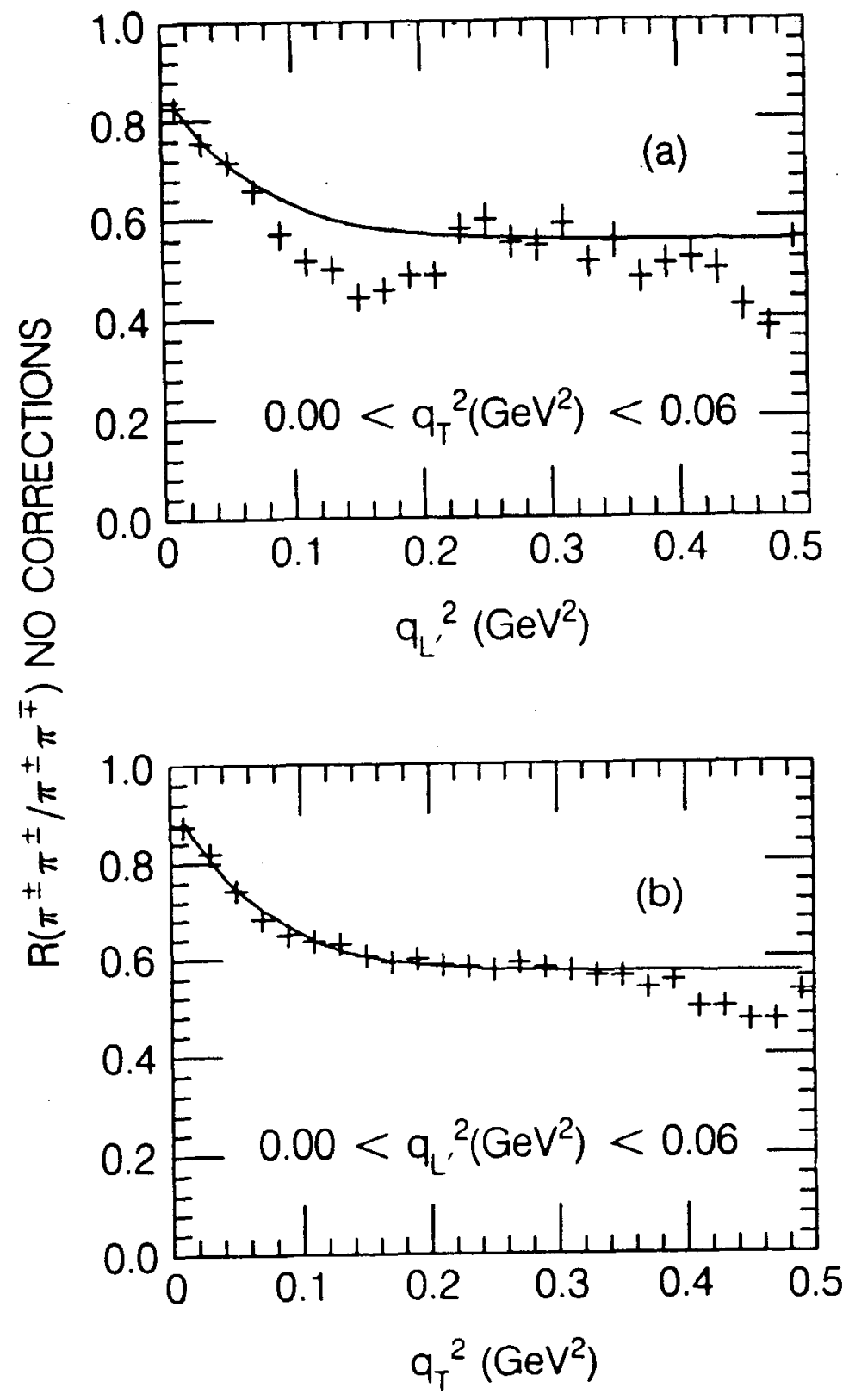

Fig. 12. The Bose-Einstein enhancement in slices of $R\left(q_{T}^{2}, q_{L^{\prime}}^{2}\right)$ for the uncorrected ratio of like to unlike pairs in the SPEAR $J / \psi$ data set. The dips due to the $K^{0}$ and $\rho^{0}$ in the unlike pairs are clearly evident in (a) the slice consisting of $0.00 \mathrm{GeV}^{2}<q_{T}^{2}<0.06 \mathrm{GeV}^{2}$ projected onto the $q_{L}^{2}$, axis; and in (b) the slice $0.00 \mathrm{GeV}^{2}<q_{L^{\prime}}^{2}<0.06 \mathrm{GeV}^{2}$ projected onto the $q_{T}^{2}$ axis. 
Table 8.

Results of fits to jet axis variables in a sample consisting of two-jet events from PEP $q \bar{q}$ data. No corrections have been applied to this data. The calculation of the measured values and the statistical (first) and systematic (second) errors is analogous to the procedure followed for Kopylov variables. Values of the parameters $r_{T}, r_{L^{\prime}}, r_{0}$, and $r_{L}$ are in $f m$. We give the $\chi^{2}$ for the unlike pair analysis first and for the mixed cluster analysis second.

\begin{tabular}{|c|c|c|c|}
\hline Data Set & $\left(q_{T}^{2}, q_{L^{\prime}}^{2}\right)$ & $\left(q_{T}^{2}, q_{0}^{2}\right)$ & $\left(q_{T}^{2}, q_{L}^{2}\right)$ \\
\hline & $\lambda=0.40 \pm 0.03 \pm 0.06$ & $\lambda=0.33 \pm 0.04 \pm 0.09$ & $\lambda=0.38 \pm 0.04 \pm 0.06$ \\
PEP $q \bar{q}$ & $r_{T}=0.66 \pm 0.07 \pm 0.07$ & $r_{T}=0.61 \pm 0.11 \pm 0.04$ & $r_{T}=0.59 \pm 0.09 \pm 0.08$ \\
& $r_{L^{\prime}}=0.71 \pm 0.08 \pm 0.10$ & $r_{0}=0.33 \pm 0.08 \pm 0.06$ & $r_{L}=0.39 \pm 0.09 \pm 0.13$ \\
& $x^{2} / D O F=1.01,1.03$ & $\chi^{2} / D O F=0.94,0.94$ & $x^{2} / D O F=0.94,0.89$ \\
\hline
\end{tabular}

Table 9. (see next page)

Table 10.

The fraction of correctly identified pions $T_{\pi}$, followed by the fraction of correctly identified pion pairs $T_{\pi \pi}$ and triplets $T_{\pi \pi \pi}$. The second, third, and fourth columns contain the fractions of like charged triplets with one $(F 1)$, two $(F 2)$, and three $(F 3)$ misidentified pions.

\begin{tabular}{|c|c|c|c|c|c|c|}
\hline $\begin{array}{c}\text { Monte Carlo } \\
\text { Data Set }\end{array}$ & $\begin{array}{l}T_{\pi} \equiv \\
\left\langle\frac{\pi_{s}}{\pi}\right\rangle\end{array}$ & $\begin{array}{l}T_{\pi \pi} \equiv \\
\left\langle\frac{\pi_{1} \pi_{i}}{\pi \pi}\right\rangle\end{array}$ & $\begin{array}{c}T_{\pi \pi \pi} \equiv \\
\left\langle\frac{\pi_{1} \pi_{1} \pi_{1}}{\pi \pi \pi}\right\rangle\end{array}$ & $\begin{array}{c}F_{1} \equiv \\
\left\langle\frac{\pi, \pi, \pi /}{\pi \pi \pi}\right\rangle\end{array}$ & $\begin{array}{c}F_{2} \equiv \\
\left\langle\frac{\pi_{i} \pi, \pi /}{\pi \pi \pi}\right\rangle\end{array}$ & $\begin{array}{c}F_{3} \equiv \\
\left\langle\frac{\pi, \pi, \pi / C}{\pi \pi \pi}\right\rangle\end{array}$ \\
\hline MC SPEAR $J / \psi$ & 0.90 & 0.81 & 0.73 & 0.24 & 0.02 & $<0.01$ \\
\hline $\mathrm{MC} \mathrm{I}$ & 0.89 & 0.80 & 0.71 & 0.26 & 0.02 & $<0.01$ \\
\hline MC SPEAR $q \Phi$ & 0.90 & 0.81 & 0.72 & 0.25 & 0.02 & $<0.01$ \\
\hline MC PEP $q q$ & 0.84 & 0.72 & 0.64 & 0.30 & 0.05 & $<0.01$ \\
\hline
\end{tabular}


using the Kopylov variables. We find the same relationship between the parameters $r_{T}, r_{L^{\prime}}, r_{0}$, and $r_{L}$ as we did in the Kopylov axis analysis. Similar results have been recently observed by the AFS Collaboration ${ }^{4}$ in two-jet events produced in pp collisions at the ISR. All the $r_{T}, r_{L^{\prime}}, r_{0}$, and $r_{L}$ values found using the jet axis variables are systematically smaller than the corresponding values found with the Kopylov variables. Note, however, that the data used in the jet axis study consists of two-jet events only, while the data used in the Kopylov analysis is the entire PEP $q \bar{q}$ data set.

An alternative method of examining source shape is to study two-jet events in the PEP $q \bar{q}$ data using the parameterization for $R\left(Q^{2}\right)$ with the replacement

$$
r \equiv \frac{r^{\prime}}{\sqrt{\sin ^{2} \theta+\cos ^{2} \theta / \kappa^{2}}}
$$

where $\theta$ is the angle between the sphericity axis of the event and the three-momentum difference in the pair rest frame ${ }^{7}$. This method is closely related to the jet axis analysis discussed above, since $Q^{2}=q_{T}^{2}+q_{L}^{2}$, is just the three-momentum difference in the pair rest frame, and $q_{T}^{2}$ and $q_{L^{\prime}}^{2}$ are components with respect to the jet axis. The parameterization in terms of $r^{\prime}$ and $\kappa$ describes a source dimension along the sphericity axis which is a factor of $\kappa$ larger (if $\kappa>1$ ) than the dimension perpendicular to the sphericity axis (assuming azimuthal symmetry). We find $r^{\prime}=0.63 \pm 0.08 \mathrm{fm}$ and $\kappa=1.49 \pm 0.55$, indicative of an ellipsoidal shape but consistent within errors with a spherical source, which is similar to the TPC result ${ }^{7}$.

\section{V.6 OTHER TESTS}

Some groups have tried pair analysis by fitting other expressions to the ratio of study to reference sample. A comparison of several fitting expressions is shown in Table 9 for the case of the uncorrected $\operatorname{SPEAR} J / \psi$ data, which has the highest statistics of all the four data sets. We find that the expression we have chosen ( (3) in the table ) describes the data as well if not better than the others listed. It has been suggested $^{3}$ that expression (5), which involves two Gaussians, is a better description 


\section{Table 9.}

The results of fitting to various expressions for $R$ for uncorrected SPEAR $J / \psi$ data. The errors shown are statistical.

\begin{tabular}{|c|c|}
\hline FITTING EXPRESSION & FIT VALUES \\
\hline $\begin{array}{l}\text { (1) } N\left(1+\lambda e^{-r^{2} Q^{3}}\right) \\
\text { no normalisation }\end{array}$ & $\begin{array}{c}\lambda=0.70 \pm 0.02 \\
r=0.76 \pm 0.02 f m \\
N=0.58 \pm 0.01 \\
\chi^{2} / D O F=58 / 54\end{array}$ \\
\hline (2) $N\left(1+\lambda e^{-r^{2} Q^{2}}\right)$ & $\begin{array}{c}\lambda=0.70 \pm 0.02 \\
r=0.76 \pm 0.02 f m \\
N=0.98 \pm 0.01 \\
\chi^{2} / D O F=58 / 54\end{array}$ \\
\hline (3) $N\left(1+\delta Q^{2}\right)\left(1+\lambda e^{-r^{2} Q^{3}}\right)$ & $\begin{array}{c}\lambda=0.69 \pm 0.03 \\
r=0.77 \pm 0.02 f m \\
N=0.99 \pm 0.01 \\
\delta=-0.01 \pm 0.02 \\
\chi^{2} / D O F=57 / 53\end{array}$ \\
\hline (4) $N\left(1+\lambda e^{-r Q}\right)$ & $\begin{array}{c}\lambda=1.19 \pm 0.03 \\
r=1.11 \pm 0.05 f m \\
N=0.95 \pm 0.01 \\
x^{2} / D O F=119 / 54\end{array}$ \\
\hline $\begin{array}{c}\text { (5) } N\left(1+\lambda_{1} e^{-r_{1}^{2} Q^{3}}+\lambda_{2} e^{-r_{2}^{2} Q^{2}}\right) \\
\lambda_{1} \text { and } \lambda_{2} \text { depend on } \\
\text { initial values used in fit, } \\
\text { while their sum is constant }\end{array}$ & $\begin{array}{c}\lambda_{1}+\lambda_{2}=0.69 \pm 0.02 \\
r_{1}=0.76 \pm 0.05 \mathrm{fm} \\
r_{2}=0.76 \pm 0.13 \mathrm{fm} \\
N=0.98 \pm 0.01 \\
\chi^{2} / D O F=58 / 52\end{array}$ \\
\hline
\end{tabular}


of $R$ in the region of small $Q^{2}$. We find that expression (5) gives essentially the same information as expression (3).

Furthermore we have also studied $R\left(Q^{2}\right)$ as a function of $\gamma$ (defined in section $\mathrm{I}$ ) for pion pairs in PEP $q \bar{q}$ data. Such a study has also, very recently, been advocated by L.S.Osborne. ${ }^{38}$ Distributions of like and unlike pairs were made for three regions of $\gamma: 1.0$ to $2.0,2.0$ to 3.4 , and greater than 3.4 , dividing the data into three sets with roughly equal statistics. We note that pion misidentification is about $10 \%$ better in the lowest $\gamma$ region than in the highest one, and that the fitted $\lambda$ 's differ slightly until the pion misidentification correction is made. After all corrections are applied, the fitted $\lambda$ 's and $r$ 's are the same within errors in the three regions of $\gamma$ and are consistent with the fully corrected measurements for PEP $q \bar{q}$ data listed in Table 4.

\section{V.7 The EFFect of Long Lived Resonances AND Particles}

The distance $c \tau$ is a measure of the pathlength at which pions from "long lived" resonances such as the $K^{*}, \omega$, and $\eta$ are produced. This distance is large compared with the effective source radius $r$ we observe. Here $c \tau=\frac{\hbar c}{\Gamma}$ where $\Gamma$ is the width of the resonance. It was thus expected ${ }^{39}$ that dipions in which one pion comes from the decay of the resonance while the other comes from the remainder of the event will correspond to separations of many fm. The Bose-Einstein effect will thus tend enhance very low $Q^{2}$ regions. As mentioned above such regions are not accessible to our experiment. Thus the end result would be that the effect of resonances will give rise to a decrease in $\lambda$. From a crude estimate we obtain a decrease of about $35 \%$. Here again, as in the case of final state interactions mentioned above, correcting for this effect would increase $\lambda$ significantly above unity for two of our data sets, namely the SPEAR $J / \psi$ and PEP $\gamma \gamma$ data. A very similar conclusion was obtained by the $\mathrm{CLEO}^{8}$ experiment.

On the other hand, the SPEAR $q \bar{q}$ and PEP $q \bar{q}$ data sets show a significantly smaller $\lambda$. The major difference between the SPEAR $J / \psi, \operatorname{PEP} \gamma \gamma$ data sets and the SPEAR $q \bar{q}$, PEP $q \bar{q}$ data sets, in terms of hadronic production, is the presence 
of charmed mesons in SPEAR $q \bar{q}$ data and charmed and bottom mesons in PEP $q \bar{q}$ data. We have used Monte Carlo simulated events to estimate how large a suppression we can expect from the presence of charmed and bottom mesons.

In order to make this estimate, we analyze Monte Carlo data using the same cuts as for the actual data. We model the Bose-Einstein correlation in the Monte Carlo by weighting the like charged pairs with the expression for $R\left(Q^{2}\right)$ and using $(\lambda, r)=(1.00,0.8 \mathrm{fm})$ as input. We weight only pairs of pions which are correctly identified as pions, so the resulting distribution corresponds to data distributions which have been fully corrected ${ }^{40}$. The PEP $q \bar{q}$ data set contains both charmed and bottom mesons. To model these data we weight all like charged Monte Carlo pairs of correctly identified pions except pairs consisting of decay products of charmed and bottom mesons. (Pairs of like pions coming from the same meson are still weighted). Fitting the ratio of weighted like to unlike Monte Carlo data pairs, we get (statistical errors only)

$$
\begin{gathered}
\lambda=0.63 \pm 0.03 \\
r=0.81 \pm 0.04 \mathrm{fm} \\
\delta=0.16 \pm 0.02 \mathrm{GeV}^{-2}
\end{gathered}
$$

Thus $\lambda$ is reduced to $\approx 60 \%$ of its maximum theoretical value due to the presence of charm and bottom mesons, while $r$ is essentially unaffected. Comparing these results with the fitted parameters in the fully corrected PEP $q \bar{q}$ data set (Table 4),

$$
\begin{gathered}
\lambda=0.50 \pm 0.04 \\
r=0.84 \pm 0.06 \mathrm{fm} \\
\delta=0.23 \pm 0.03 \mathrm{GeV}^{-2},
\end{gathered}
$$

we see that the presence of charmed and bottom mesons seems to account for the major fraction of the suppression of $\lambda$ from its maximum value. An analogous study of Monte Carlo data representing the SPEAR $q \bar{q}$ data set, which contains charmed 
but no bottom mesons, similarly gives a suppressed value of $\lambda=0.66 \pm 0.03$. From Table 4 we see that the measured (fully corrected) value of $\lambda$ for the SPEAR $q \bar{q}$ data is very close: $\lambda=0.66 \pm 0.04$.

These studies indicate that once charmed and bottom mesons are corrected for, very little room remains for possible source coherence effects or for other unaccounted corrections. 


\section{SUMMARY AND CONCLUSIONS}

We have studied the Bose-Einstein effect for both pairs of like pions and triplets of like pions. Our study extended over four data sets obtained with the Mark II detector at the Stanford Linear Accelerator Center. The four sets differ both in energy and in pion production mechanism and include runs both at SPEAR and at PEP.

Figure 13 displays the values of $\lambda$ and $r$ for the pair analysis of the four data sets, arranged roughly in the order of increasing energy available for hadron production. We find that after all corrections have been applied, the SPEAR $J / \psi$ and PEP $\gamma \gamma$ data sets show nearly maximal values of $\lambda$. If we accept the concept implied by Bose-Einstein statistics that the maximum value for $\lambda$ is $\lambda=1$ then we find that there is no room for the following two effects which tend to decrease $\lambda$. A decrease of $\approx 35 \%$ in $\lambda$ is expected due to the effect of long lived resonances such as the $K^{*}, \omega$, and $\eta$. Like $\mathrm{CLEO}^{8}$, we find that correcting for the effect of resonances in these two data sets would bring $\lambda$ above its maximum expected value. Similarly, no significant suppression due to the effects of the final state strong interaction is evident within errors in these two data sets. Given a larger statistical sample at the $J / \psi$, such as the data collected by the MARK III at SPEAR or the DM2 at DCI ORSAY, it would be interesting to see whether pairing pions from the $\omega$ or other resonance region with pions outside such regions affects $\lambda$ to any greater degree than our result indicates.

The SPEAR $q \bar{q}$ and PEP $q \bar{q}$ data sets show significantly smaller values of $\lambda$. In the previous section we saw that a major fraction of this suppression in $\lambda$ can be accounted for by the presence of charmed mesons in the SPEAR $q \bar{q}$ data and both charmed and bottom mesons in the PEP $q \bar{q}$ data, leaving only a small part of the suppression for other possible effects, such as source coherence. Although we are unable to do the analysis because of low statistics, it would again be interesting to study the Bose-Einstein effect in charm and bottom enriched events.

The values of the parameter $r$ appear to be fairly constant $(\approx 0.7 \mathrm{fm})$ for all four data sets, as well as for $e^{+} e^{-}$data from 10 to $34 \mathrm{GeV}^{7,8,9}$. It is this 

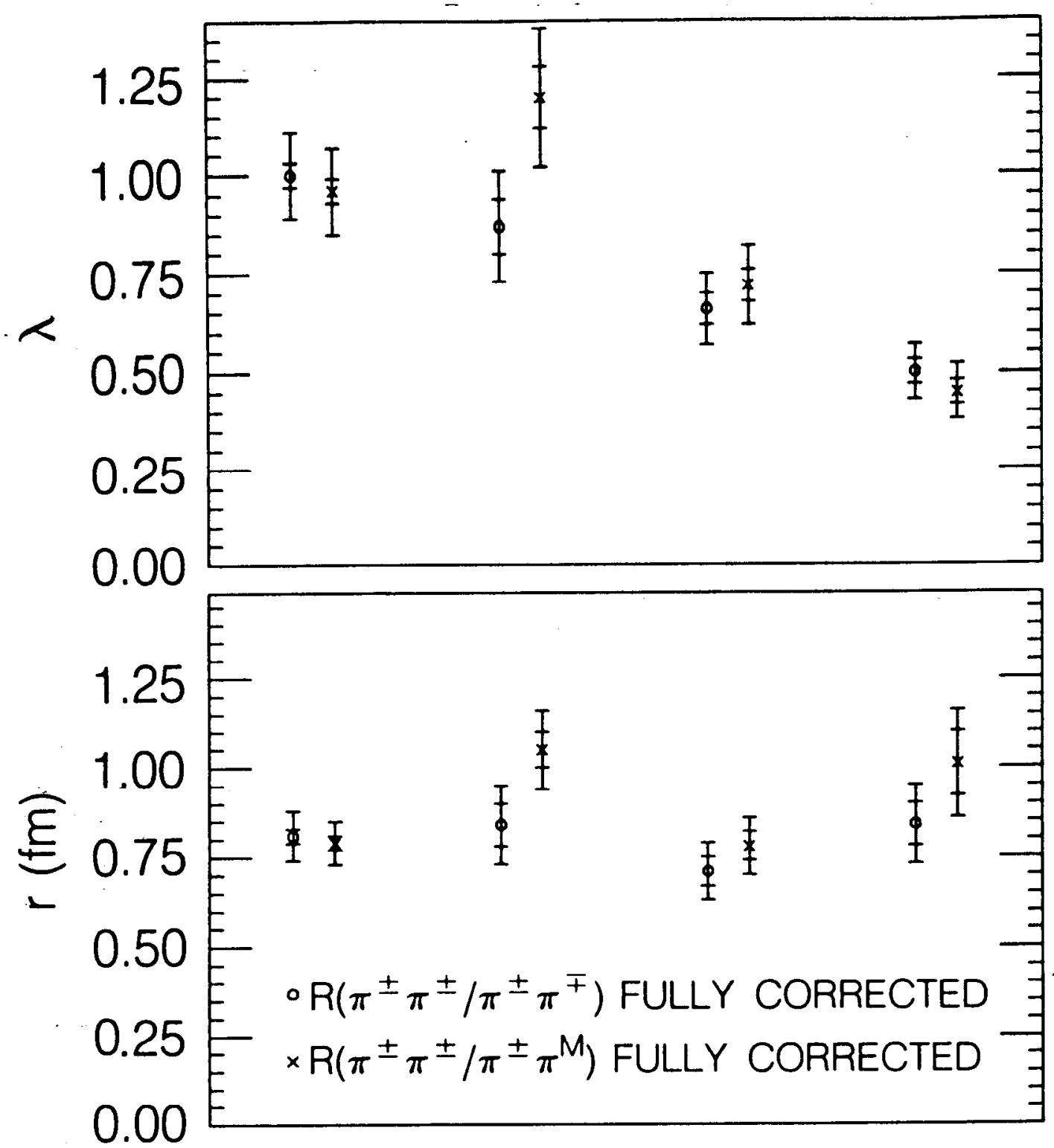

SPEAR J/ $\psi$ PEP $\gamma \gamma \quad$ SPEAR $q \bar{q} \quad$ PEP $q \bar{q}$

Fig. 13. Plots of $\lambda$ and $r$ for the four data sets, arranged roughly in the order of increasing energy available for hadron production. The values shown are for the fully corrected ratio of like to unlike charged pair distributions (circles) and of like to mixed cluster pair distributions (stars). The errors indicated are statistical (inner bars) and systematic (difference between inner and outer bars). 
remarkable constancy over such very different data sets which leads us to believe that the idea that $r$ represents the size of the local region, responsible for the pion pairs studied, rather then the size of the entire source, ${ }^{22}$ may be correct. This result is to be contrasted with the relativistic heavy ion data where a fireball model or the superposition of several color strings is apropriate and where indeed much larger radii, ${ }^{5,6}$ which appear to correspond to the entire source, have been observed.

Results of the triplet analysis are summarized in Figure 14, which displays the values of $\lambda_{3}$ and $r_{3}$ found using the mixed cluster reference sample. Although statistics are more limited in triplet analysis than in pair analysis, overall we find qualitatively similar patterns in $\lambda_{3}$ and $r_{3}$ as we do in $\lambda$ and $r$. The SPEAR $J / \psi$ data set, with the largest statistics, clearly shows nearly maximum value of $\lambda_{3}$ using the mixed cluster reference sample with all corrections. The value of $r_{3}$ is approximately the same for all the data sets, and $r_{3}^{2}$ is between $1 / 3$ and $1 / 2$ of the value of $r^{2}$, as expected from consistency arguments between pair and triplet parameterizations.

We have studied $\lambda$ and $r$ as a function of $p_{T \max }$ in the PEP $\gamma \gamma$ data, and a summary of the results is shown in Figure 15. Within the available statistics, we find that $\lambda$ in the lowest $p_{T \text { max }}$ bin (VDM region) is consistent with maximum value, and that $\lambda$ decreases with increasing $p_{T \max }$ (transition to hard scattering region, where again charm production is expected).

A study of the pion pair Bose-Einstein enhancement in the two-dimensional distribution of invariant quantities $R\left(q_{T}^{2}, q_{L^{\prime}}^{2}\right)$ gives the same values for the parameters $r_{T}$ and $r_{L^{\prime}}$, both when $q_{T}$ is calculated with respect to the net pair three-momentum (Kopylov axis) and when it is calculated with respect to the jet axis for two-jet events in the PEP $q \bar{q}$ data set. Similar results have been reported by CLEO $^{8}$, TASSO $^{9}$, and $\mathrm{AFS}^{3}$. Fits to the two-dimensional distributions of non-invariant quantities $R\left(q_{T}^{2}, q_{L}^{2}\right)$ and $R\left(q_{T}^{2}, q_{0}^{2}\right)$ indicate that $r_{0} \approx r_{L} \approx \frac{2}{3} r_{T}$ to $\frac{1}{2} r_{T}$.

Here $r_{L^{\prime}} \approx r_{T}$ implies that the "local" source region for the pions studied is nearly spherical in the di-pion rest frame. One can interpret the fact that $\tau_{L}<r_{L^{\prime}}$ 


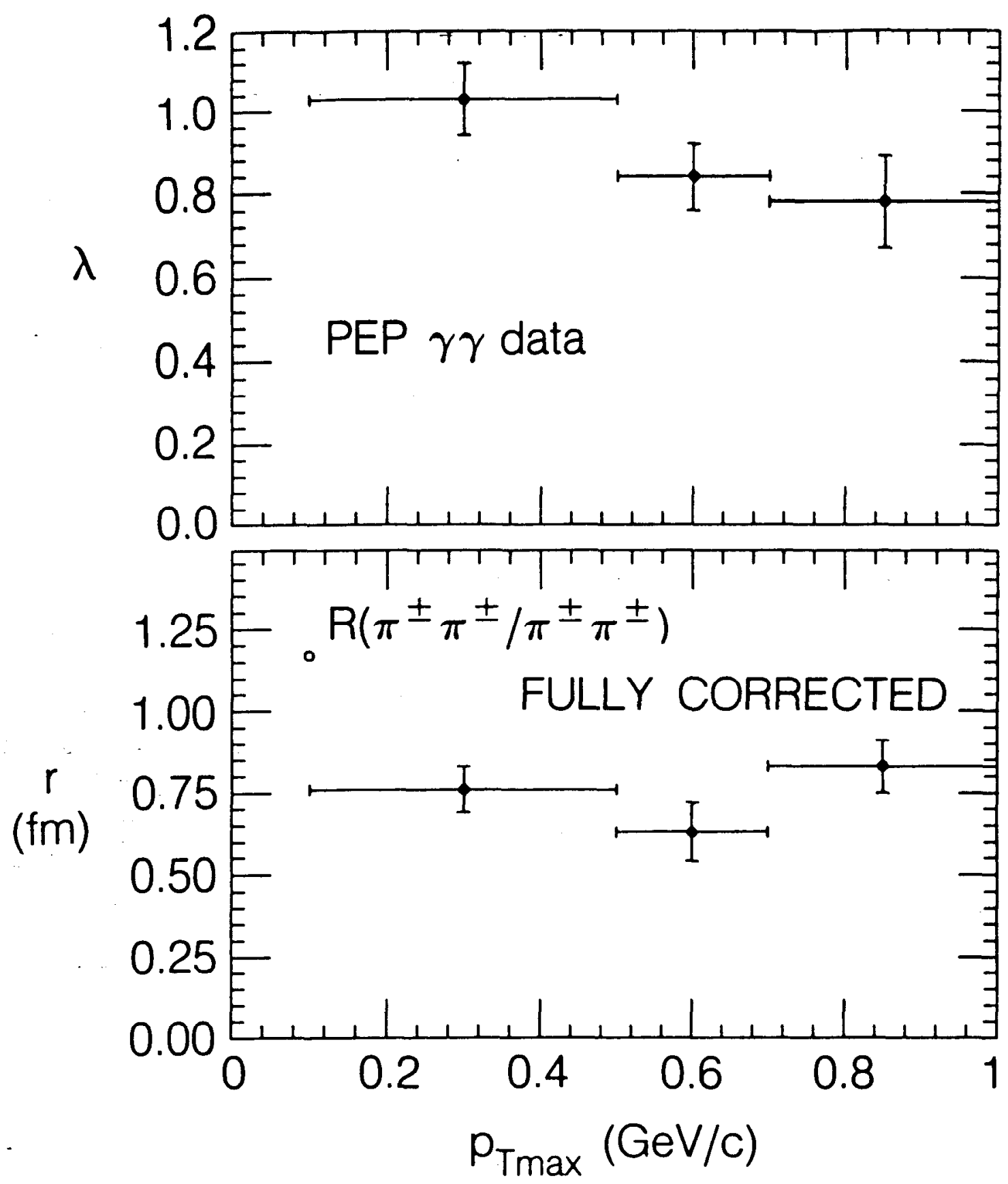

Fig. 14. Plots of $\lambda_{3}$ and $r_{3}$ for the four data sets, arranged roughly in the order of increasing energy available for hadron production. The values shown are for the fully corrected ratio of like to mixed cluster triplet distributions. The errors indicated are statistical (inner bars) and systematic (difference between inner and outer bars). 

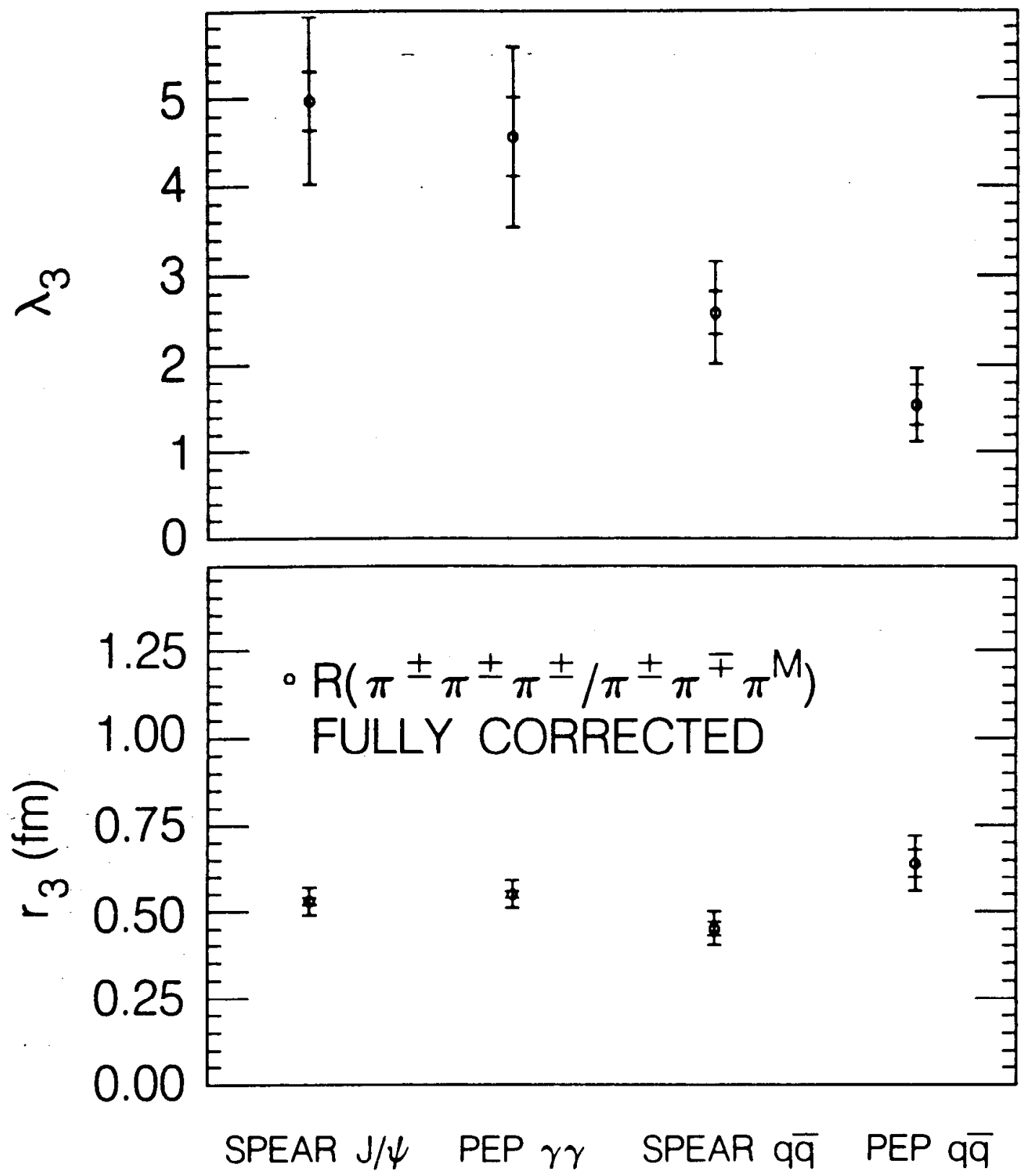

F士g. 15. Plots of $\lambda$ and $r$ for the PEP $\gamma \gamma$ data set as a function of $p_{\text {Tmax }}$. The values shown are for the fully corrected ratio of like to unlike pair distributions, and the errors indicated are statistical. 
in terms of an expanding local source region. In the dipion rest frame $\left(r_{L^{\prime}}\right)$ the local source moves with the dipions. In the laboratory system $\left(r_{L}\right)$ the local source appears contractred. 41 These results point to the fact that the invariant form $R\left(Q^{2}\right)$ is a good representation of the data. The non-invariant variables $r_{L}$ and $r_{0}$ in the Kopylov formulation then simply reflect the relations

$$
q_{L^{\prime}}^{2}=\frac{q_{L}^{2}}{\gamma^{2}}=\frac{q_{0}^{2}}{\gamma^{2}-1}
$$

and hence $q_{L} \approx q_{0}$, without adding additional information. This is in agreement with the conclusions of the CLEO ${ }^{8}$ and $\operatorname{TASSO}^{9}$ collaborations.

\section{ACKNOWLEDGEMENTS}

We wish to thank M. Gyulassy, W. Hofmann, and M. Suzuki for useful discussions regarding current interpretations of the Bose-Einstein correlation parameters. 


\section{Appendix A. COULOMB CORRECTION}

For like (l) and unlike ( $\mathrm{u}$ ) charged pairs the inclusive distribution in the presence of the pair Coulomb interaction is given by

$$
\begin{gathered}
P_{l}\left(k_{1}, k_{2}\right)=G_{l}(\eta) P_{n}\left(k_{1}, k_{2}\right), \\
P_{u}\left(k_{1}, k_{2}\right)=G_{u}(\eta) P_{n}\left(k_{1}, k_{2}\right),
\end{gathered}
$$

where $P_{n}\left(k_{1}, k_{2}\right)$ is the pair inclusive distribution in the absence of the Coulomb force, and

$$
\begin{aligned}
G_{l}(\eta) & =\frac{2 \pi \eta}{\exp (2 \pi \eta)-1}, \\
G_{u}(\eta) & =\frac{2 \pi \eta}{1-\exp (-2 \pi \eta)}, \\
\eta & =\frac{\alpha m_{\pi}}{\left|k_{1}-k_{2}\right|} .
\end{aligned}
$$

Here, $\alpha=1 / 137$, and $k_{1}$ and $k_{2}$ are pion four-momenta in the pair center of mass frame, so that

$$
\eta=\frac{\alpha m_{\pi}}{\sqrt{Q^{2}}}
$$

The correction for Coulomb effects is done by weighting each like pair by $1 / G_{l}(\eta)$ and each unlike pair by $1 / G_{u}(\eta)$. The ratio of the corrected like to corrected unlike pairs then represents $R$ in the absence of the Coulomb interaction. The correction is small except for very small $Q^{2}$ values, as shown in Fig. 16.

The extension of this correction to charged pion triplets was derived by $\mathrm{Liu}^{37}$ to first order in $\alpha$. If we label the three pions in a triplet as 1,2 , and 3 , then the charged triplet distribution $P_{c}\left(k_{1}, k_{2}, k_{3}\right)$ is related to the same distribution in the absence of the Coulomb interaction $P_{n}\left(k_{1}, k_{2}, k_{3}\right)$ as follows:

$$
P_{c}\left(k_{1}, k_{2}, k_{3}\right)=G\left(\eta_{12}\right) G\left(\eta_{23}\right) G\left(\eta_{31}\right) P_{n}\left(k_{1}, k_{2}, k_{3}\right),
$$

where

$$
G\left(\eta_{i j}\right)= \begin{cases}G_{l}\left(\eta_{i j}\right), & \text { for a like charged pair } \\ G_{u}\left(\eta_{i j}\right), & \text { for an unlike charged pair }\end{cases}
$$


GAMOW FACTORS

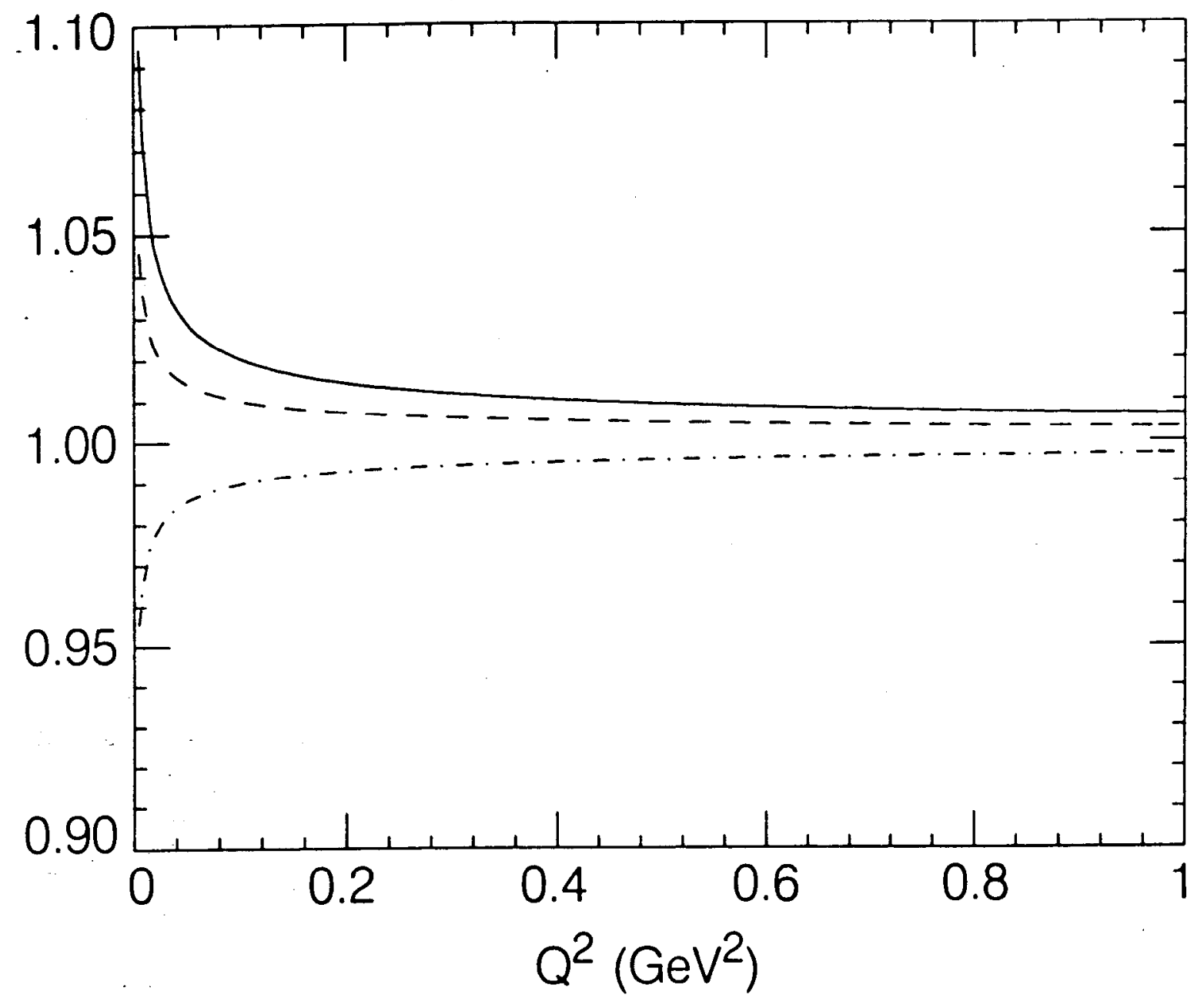

Fig. 16. The Gamow factors $G_{l}(\eta)$ (dot-dash line) and $G_{u}(\eta)$ (dashed line). In order to get the ratio $R$ of like to unlike charged pairs in the absence of the Coulomb final state interaction, we in effect multiply the observed $R$ by the factor $G_{u} / G_{l}$ (solid line). 
We use three kinds charged triplets. The like charged triplet contains three like charged pairs, so the Gamow correction is given by

$$
C_{1} \equiv G_{l}\left(\eta_{12}\right) G_{l}\left(\eta_{23}\right) G_{l}\left(\eta_{31}\right)
$$

The $\pm \pm \mp$ reference sample triplet contains one like charged pair and two unlike charged pairs, so this reference sample is corrected using

$$
C_{2} \equiv G_{l}\left(\eta_{12}\right) G_{u}\left(\eta_{23}\right) G_{u}\left(\eta_{31}\right)
$$

Finally, the mixed cluster reference sample triplet contains one unlike charged pair and two effectively neutral pairs, so this reference sample is corrected with

$$
C_{3} \equiv G_{u}\left(\eta_{12}\right)
$$

In analogy to the procedure in pairs, the Coulomb correction for triplets is done by weighting each like charged triplet with $1 / C_{1}$, each $\pm \pm \mp$ triplet with $1 / C_{2}$, and each mixed cluster reference triplet with $1 / C_{3}$. 


\section{Appendix B. PION MISIDENTIFICATION CORRECTION}

Some fraction of the study sample consists of pairs in which one or both particles have been misidentified as pions. Such pairs exhibit no Bose-Einstein correlation, and to a first approximation we can model their distribution in $Q^{2}$ using the distribution of reference pairs. The contamination of reference pairs by misidentified pions is neglected. An analogous treatement is applied to the triplet study sample.

Let us define $T_{\pi \pi}$ as the fraction of like charged pairs containing two correctly identified pions. The pion misidentification correction is done bin by bin on the like charged pion pair distribution in $Q^{2}$ as follows:

$$
B_{l}^{\text {corr }}=B_{l}-\frac{n_{l}}{n_{u}} B_{u}\left(1-T_{\pi \pi}\right)
$$

where

- $B_{l}$ and $B_{u}$ are the bin contents of the like charged pair distribution and the unlike charged pair distribution, respectively,

$-n_{l}$ and $n_{u}$ are the number of like and unlike charged pairs, respectively, in the region $0.68 \mathrm{GeV}^{2}<Q^{2}<1.0 \mathrm{GeV}^{2}$. We use this region to normalize the number of like pairs to the number of unlike pairs.

The fraction $T_{\pi \pi}$ is listed in Table 10 for each of the four data sets.

Like charged triplets contain a fraction $T_{\pi \pi \pi}$ of three correctly identified pions, a fraction $F 1$ of two correct and one misidentified pion, a fraction $F 2$ of one correct and two misidentified pions, and a fraction $F 3$ of three misidentified pions. The relative size of these fractions is listed in Table 10. Fractions $F 2$ and $F 3$ exhibit no Bose-Einstein correlation and are therefore modeled by the mixed cluster reference sample. Fraction $F 1$ contains one like charged pion pair and is modeled by the $\pm \pm \mp$ reference sample.

Analogous to pairs, the pion misidentification correction is done bin by bin on 
like triplet $Q_{3}^{2}$ distributions as follows:

$$
B_{l}^{\text {corr }}=B_{l}-\frac{n_{l}-}{n_{ \pm \pm \mp}} B_{ \pm \pm \mp} F_{1}-\frac{n_{l}}{n_{m}} B_{m}\left(F_{2}+F_{3}\right)
$$

where

$-B_{l}, B_{ \pm \pm \mp}$, and $B_{m}$ are the bin contents of the like charged, $\pm \pm \mp$, and mixed cluster triplet distributions respectively,

- $n_{l}, n_{ \pm \pm \mp}$, and $n_{m}$ are the relative numbers of like charged, $\pm \pm \mp$, and mixed cluster triplets in the region of $0.5 \mathrm{GeV}^{2}<Q_{3}^{2}<1.0 \mathrm{GeV}^{2}$.

The pion misidentification correction is carried out after the appropriate Coulomb final state correction has been applied to each distribution. 


\section{Appendix C. MIXED CLUSTER REFERENCE SAMPLES}

The goal of event mixing is to produce a reference sample which is free of undesirable correlations (such as resonances in unlike charged pairs and the BoseEinstein correlation in the $\pm \pm \mp$ triplets) while reproducing the kinematical phase space of the study sample. The most common event mixing procedure pairs all the pions from one event with all the pions from another event. For event topologies containing clear jets or clusters, such as our PEP $q \bar{q}$ and PEP $\gamma \gamma$ data sets, this algorithm produces pairs whose kinematical phase space distribution is distorted and therefore cannot be used as as a reference sample. Our event mixing algorithm, referred to as cluster mixing, reproduces the kinematical phase space of the study samples in all four data sets by a careful combination of parts of events commonly called clusters.

Events in each of the four data sets are passed through a Lund Monte Carlo cluster finding routine, which defines clusters of particles using all the "good quality" momentum vectors of both charged and neutral particles in an event. This routine assigns the input particles to the found clusters, and labels the clusters in each event in order of decreasing cluster momentum (assigns the cluster order number). The SPEAR $J / \psi$, SPEAR $q \bar{q}$, and PEP $\gamma \gamma$ data sets are made up almost entirely of one-cluster events, although the typical cluster in a PEP $\gamma \gamma$ event is more "bunched up" than in the other two data sets. The PEP $q \bar{q}$ data consists mostly of two-cluster events (69\%) and events with three or more clusters.

The cluster mixing algorithm takes the event being analyzed (analysis event) and creates a modified event by the following procedure: to each cluster in the analysis event, the cluster mixing algorithm adds on the momentum vectors of a selected set of pions $\pi^{M}$ from an appropriate cluster of another event. The criteria for the selection of the pions $\pi^{M}$ are based on the nature of the cluster to which they are to be added (called the analysis cluster). First, the multiplicity of $\pi^{M}$ is chosen to be the average of the $\pi^{+}$and $\pi^{-}$multiplicities in the analysis cluster. Second, the $\pi^{M}$ are required to originate from a cluster of the same order number as the analysis cluster. In a two-jet event, for example, $\pi^{M}$ added to the lower energy 
jet must come from the lower energy jet of another two-jet event. Finally, we define a net three-momentum of the analysis cluster by summing the three-momenta of all the pions and other charged and neutral tracks (called non-pions) used to find the cluster. The three-momentum vectors of $\pi^{M}$ are then rotated as a group, so that the net three-momentum of the $\pi^{M}$ is parallel to the net three-momentum of all the non-pion particles in the analysis cluster.

The modified event, then, consists of the original $\pi^{+}$and $\pi^{-}$and the added $\pi^{M}$. The modified event is assembled cluster by cluster from the analysis event, but the reference pairs and triplets in the modified event are made using the modified event as a whole. Since the $\pi^{M}$ are not correlated with either the $\pi^{+}$or the $\pi^{-}$ in the modified event, the mixed cluster pair reference sample $\pi^{ \pm} \pi^{M}$ contains no resonance signals, and the mixed cluster triplet reference sample $\pi^{+} \pi^{-} \pi^{M}$ is entirely free of the Bose-Einstein correlation. 


\section{REFERENCES}

1. For recent reviews and additional references see: W. A. Zajc, Hadronic Multiparticle Production, Peter Carruthers, ed., World Scientific Book, (1988); G. Goldhaber, Proc. of the First Int. Workshop on Local Equilibrium in Strong Interaction Physics, D. K. Scott and R. M. Weiner, ed., World Scientific Publishing Co. (1985); G. Goldhaber and I. Juricic, Proc. of the Second Int. Workshop on Local Equilibrium in Strong Interaction Physics, P. Carruthers and D. Strottman, ed., World Scientific Publishing Co. (1986).

2. M. Deutschmann et. al., Nucl. Phys. B 204, 333 (1982).

3. T. Akesson et al.,Phys. Lett. B 187420 (1987).

4. B. Lörstad (AFS Collaboration), XVIII Int. Symp. on Multiparticle Dynamics, Tashkent, USSR 1987.

5. W. A. Zajc, Phys. Rev. C 29, 2173 (1984).

6. T. J. Humanic (NA35 Collaboration), Quark Matter 1987 Conf., Nordkirchen, West Germany, 23-29 August 1987.

7. H. Aihara et al. (TPC Collaboration), Phys. Rev. D 31, 996 (1985).

8. P. Avery et al. (CLEO Collaboration), Phys. Rev. D 32, 2294 (1985).

9. M. Althoff et al. (TASSO Collaboration), Z. Phys. C 30, 355 (1986).

10. G. Gidal (MARK II Collaboration), Proc. of the VIIth Int. Workshop on Photon Photon Collisions, World Scientific Publishing Co., (April 1986).

11. M. Arneodo et. al. (EMC Collaboration), Z. Phys. C 32, 1, (1986).

12. G. Goldhaber et al., Phys. Rev. Lett. 3, 181 (1959).

13. G. Goldhaber, S. Goldhaber, W. Lee, and A. Pais, Phys. Rev. 120, 300 (1960).

14. R. Hanbury-Brown and R. Q. Twiss, Nature 178, 1046 (1956).

15. G. Cocconi, Phys. Lett. 49B, 459 (1974).

16. E. V. Shuryak, Phys. Lett. 44B, 387 (1973).

17. M. Gyulassy, S. K. Kauffmann and L. W. Wilson, Phys.Rev. C 20,2267 (1979). 
18. G. N. Fowler and R. M. Weiner, Phys. Lett. 70B, 201 (1977).

19. A. Giovannini and G. Veneziano, Nucl. Phys. B 130, 61 (1977).

20. M. G. Bowler, Z. Phys. C 29, 617 (1985).

21. B. Andersson and W. Hofmann, Phys. Lett. 169B, 364 (1986).

22. W. Hofmann, pres. at the Workshop on Electronuclear Physics with Internal Targets, Stanford, CA January 5-8, 1987 (LBL-23108).

23. X. Artru and M. G. Bowler, Oxford-NP 79/86 (1986), submitted to Z. Phys C.

24. M. G. Bowler, Phys. Lett. 180B, 299 (1986).

25. G. Goldhaber, Proc. of the Int. Conf. on High Energy Physics, Lisbon, Portugal, July 9-15, 1981, J. Dias de Deus and J. Soffer, ed., European Physical Society, (1982).

26. G. I. Kopylov and M. I. Podgoretskii, Sov. J. Nucl. Phys. 15, 219 (1972).

27. R. H. Schindler, et al., Phys. Rev. D 24, 78 (1981).

28. J. Jaros, Proc. of the Int. Conf. on Instrumentation for Colliding Beam Physics, W. Ash, ed., SLAC-Report 250, (1982).

29. The number of jets in the event is found by a MARK II utility program called JETCAL, which uses sphericity and sphericity-like variables in its calculations.

30. M. E. Nelson, Ph.D. Thesis, University of California, Berkeley, LBL-16724, (1983), (unpublished).

31. The TOF weight for a given mass hypothesis is defined as $W=(1 / N) \exp ((t-$ $\left.t_{m}\right)^{2} / 2 \sigma^{2}$, where $t$ is the measured time of flight, $\sigma$ is the measured TOF resolution, and $t_{m}$ is the expected time of flight $t_{m} \equiv(l / p c) \sqrt{p^{2}+m^{2}}$ for a particle of mass $m$, momentum $p$, and path length $l$. The normalization $N$ is the sum of weights over three hypotheses: pion, kaon, and proton.

32. M. Suzuki, Phys. Rev. D 35, 3359 (1987).

33. M. G. Bowler, Oxford preprint OUNP 65/87 (1987).

34. A. S. Davydov, Quantum Mechanics, trans., ed., and with additions by 
D. ter Haar, Pergamon Press, London (1965); (section 100, p.403).

35. I. Juricic, Ph.D. Thesis, University of California, Berkeley, (1987), LBL24493, (unpublished).

36. F. James and M. Ross, MINUIT, CERN program library D506/D516.

37. Y. Liu, Ph.D. Thesis, University of California, Riverside, (1985), (unpublished).

38. L. S. Osborne Phys. Rev. Lett. 60, 987 (1988).

39. P. Grassberger, Nucl. Phys. B102, 221 (1977)

40. Monte Carlo data does not simulate the final state Coulomb interaction, so no correction is required for that effect here.

41. See for example, Y. Hama and S. S. Padula IFUSP/P 673 (1987) Phys. Rev. $\mathbf{D}$ to be published. 